Article

\title{
Strong Convergence Theorems for Fixed Point Problems for Nonexpansive Mappings and Zero Point Problems for Accretive Operators Using Viscosity Implicit Midpoint Rules in Banach Spaces
}

\author{
Huancheng Zhang ${ }^{1, *}$, Yunhua $\mathrm{Qu}^{2}$ and Yongfu $\mathrm{Su}^{3}$ \\ 1 Qinggong College, North China University of Science and Technology, Tangshan 063000, China \\ 2 Qian'an College, North China University of Science and Technology, Tangshan 064400, China; \\ yunhuaqu@163.com \\ 3 Department of Mathematics, Tianjin Polytechnic University, Tianjin 300387, China; tjsuyongfu@163.com \\ * Correspondence: zhanghuancheng521@163.com; Tel.: +86-189-3157-1573
}

Received: 13 October 2018; Accepted: 14 November 2018; Published: 16 November 2018

check for updates

\begin{abstract}
This paper uses the viscosity implicit midpoint rule to find common points of the fixed point set of a nonexpansive mapping and the zero point set of an accretive operator in Banach space. Under certain conditions, this paper obtains the strong convergence results of the proposed algorithm and improves the relevant results of researchers in this field. In the end, this paper gives numerical examples to support the main results.
\end{abstract}

Keywords: viscosity implicit midpoint rule; nonexpansive mapping; accretive operator; zero point problem

\section{Introduction}

Let $E$ be a Banach Space and $E^{*}$ the dual space. $J$ denotes the normalized duality mapping from $E$ to $2^{E^{*}}$ and is defined by

$$
J(x)=\left\{f \in E^{*}:<x, f>=\|x\|^{2}=\|f\|^{2}\right\}, x \in E .
$$

Let $C$ be a nonempty set of $E$. A mapping $f: C \rightarrow C$ is contractive, if $\|f(x)-f(y)\| \leq k\|x-y\|$, $\forall x, y \in C, k \in[0,1)$. A mapping $S: C \rightarrow C$ is nonexpansive, if $\|S(x)-S(y)\| \leq\|x-y\|, \forall x, y \in C$. Let $F(S)$ denote the fixed point set of $S$.

$A: C \rightarrow E$ is called accretive operator, if there exists $j(x-y) \in J(x-y)$ such that $\langle A x-A y, j(x-y)\rangle \geq 0$, for any $x, y \in C$. If $R(I+r A)=E, \forall r>0$, then $A$ is called $m$-accretive operator. $J_{r}: R(I+r A) \rightarrow D(A)$ is called the resolvent of $m$-accretive operator $A$ and defined by $J_{r}=(I+r A)^{-1}, \forall r>0$. It is well known that $J_{r}$ is nonexpansive mapping and $N(A)=F\left(J_{r}\right)$, where $N(A)=\{x \in E: 0 \in A x\}$ and $F\left(J_{r}\right)$ is the fixed point set of $J_{r}$. So fixed point theory of nonexpansive mappings has been applied to zero point problem of accretive operator, see [1-6] and the references therein.

The implicit midpoint rule is one of the powerful methods for solving ordinary differential equations; see [7-12] and the references therein. Moreover, viscosity iterative algorithms for finding common fixed points for nonlinear operators and solutions of variational inequality problems have been researched by many authors. 
In 2009, Chang et al. [1] proposed a viscosity iterative algorithm for accretive operator and nonexpansive mapping:

$$
\left\{\begin{array}{c}
x_{0}=x \in C, \\
y_{n}=\beta_{n} x_{n}+\left(1-\beta_{n}\right) S J_{r} x_{n}, \\
x_{n+1}=\alpha_{n} f\left(x_{n}\right)+\left(1-\alpha_{n}\right) y_{n}, \forall n \geq 0 .
\end{array}\right.
$$

In 2010, Jung [13] proposed a composite iterative algorithm by viscosity method for finding the zero point of an accretive operator:

$$
\left\{\begin{array}{c}
x_{0}=x \in C \\
y_{n}=\beta_{n} x_{n}+\left(1-\beta_{n}\right) J_{r_{n}} x_{n} \\
x_{n+1}=\alpha_{n} f\left(x_{n}\right)+\left(1-\alpha_{n}\right) y_{n}, \forall n \geq 0 .
\end{array}\right.
$$

In 2016, Jung [14] extended the related results and proposed an iterative algorithm for finding common point of zero of accretive operator and fixed point of nonexpansive mapping:

$$
\begin{gathered}
x_{n+1}=J_{r_{n}}\left(\alpha_{n} f\left(x_{n}\right)+\left(1-\alpha_{n}\right) S x_{n}\right), \forall n \geq 0, \\
x_{n+1}=J_{r_{n}}\left(\alpha_{n} f\left(x_{n}\right)+\left(1-\alpha_{n}\right) S x_{n}+e_{n}\right), \forall n \geq 0 .
\end{gathered}
$$

In 2017, $\mathrm{Li}$ [15] introduced a new iterative algorithm in a real reflexive Banach space $E$ with the uniformly Gâteaux differentiable norm and $C$ is a nonempty closed convex subset of $E$ which has the normal structure:

$$
\left\{\begin{array}{c}
x_{0}=x \in C \\
y_{n}=\beta_{n} S J_{r_{n}}\left(e_{n}+x_{n}\right)+\left(1-\beta_{n}\right) x_{n} \\
x_{n+1}=\alpha_{n} T\left(x_{n}\right)+\left(1-\alpha_{n}\right) y_{n}, \forall n \geq 0
\end{array}\right.
$$

In 2015, Xu et al. [16] used viscosity iterative algorithm to implicit midpoint rule for nonexpansive mapping in Hilbert space and proposed viscosity implicit midpoint rule: $\left\{x_{n}\right\}$ was generated by the following

$$
x_{n+1}=\alpha_{n} f\left(x_{n}\right)+\left(1-\alpha_{n}\right) T\left(\frac{x_{n}+x_{n+1}}{2}\right), n \geq 0 .
$$

Under some conditions on $\left\{\alpha_{n}\right\}$, they obtained that $\left\{x_{n}\right\}$ strongly converged to $q \in F(T)$, and $q$ was the solution of variational inequality $\langle(I-f) q, x-q\rangle \geq 0, x \in F(T)$.

In 2017, Luo et al. [17] extended the results of Xu et al. [16] from Hilbert space to uniformly smooth Banach space: $\left\{x_{n}\right\}$ was generated by the following

$$
x_{n+1}=\alpha_{n} f\left(x_{n}\right)+\left(1-\alpha_{n}\right) T\left(\frac{x_{n}+x_{n+1}}{2}\right), n \geq 0 .
$$

Under some conditions, they obtained that $\left\{x_{n}\right\}$ strongly converged to $q \in F(T)$, and $q$ was the solution of variational inequality $\langle(I-f) q, j(x-q)\rangle \geq 0, x \in F(T)$.

Motivated and inspired by the above papers, this paper uses the viscosity implicit midpoint rule to find common points of the fixed point set of a nonexpansive mapping and the zero point set of an accretive operator in Banach space and obtains the strong convergence results and improves the previous results. Finally, this paper gives numerical examples to support the main results.

\section{Preliminaries}

For all $\varepsilon \in[0,2],\|x\|=\|y\|=1,\|x-y\| \geq \varepsilon$, if there exists $\delta_{\varepsilon}>0$ such that $\frac{\|x+y\|}{2}<1-\delta_{\varepsilon}$, then $E$ is called uniformly convex. A Banach space is uniformly convex if and only if there exists a continuous strictly increasing convex function $g:[0,+\infty) \rightarrow[0,+\infty)$ with $g(0)=0$ such that 


$$
\|\lambda x+(1-\lambda) y\|^{2} \leq \lambda\|x\|^{2}+(1-\lambda)\|y\|^{2}-\lambda(1-\lambda) g(\|x-y\|)
$$

For each $x, y \in U, U=\{x \in E:\|x\|=1\}$, if $\lim _{t \rightarrow 0} \frac{\|x+t y\|-\|x\|}{t}$ exists, then $E$ is said to be have a Gâteaux differentiable norm. If for each $y \in U$, the limit is attained uniformly for $x \in U$, then $E$ is said to be have a uniformly Gâteaux differentiable norm. It is well known that if $E$ has uniformly Gâteaux differentiable norm, then $J$ is single valued and norm-to-weak* uniformly continuous on each bounded subset of $E$, see [18].

Let $C$ be a closed convex subset of $E$. If for each bounded closed convex subset $D$ of $C$ which contains at least two points, there exists one element $x \in D$ which is not a diametral point of $D$ such that $\operatorname{diam}(D)>\sup \{\|x-y\|: y \in D\}$, where $\operatorname{diam}(D)$ is the diameter of $D$, then $C$ is said to have normal structure.

We need the following lemmas for the proof of our main results.

Lemma 1 [19]. For $\lambda, \mu>0, x \in E$, so $J_{\lambda} x=J_{\mu}\left(\frac{\mu}{\lambda} x+\left(1-\frac{\mu}{\lambda}\right) J_{\lambda} x\right)$.

Lemma 2 [20]. Let $\left\{a_{n}\right\},\left\{b_{n}\right\},\left\{c_{n}\right\}$ be three nonnegative real sequences satisfying

$$
a_{n+1} \leq\left(1-t_{n}\right) a_{n}+b_{n}+c_{n}, \forall n \geq 0,
$$

where $\left\{t_{n}\right\} \subset(0,1)$. If the following conditions are satisfied $\sum_{n=0}^{\infty} t_{n}=\infty ; b_{n}=o\left(t_{n}\right) ; \sum_{n=1}^{\infty} c_{n}<\infty$. So $\lim _{n \rightarrow \infty} a_{n}=0$.

Lemma 3 [4,21]. Let E be a real reflexive Banach space with the uniformly Gâteaux differentiable norm and C be nonempty closed convex subset of $E$ which has normal structure. Let $S: C \rightarrow C$ be a nonexpansive mapping with a fixed point and $T: C \rightarrow C$ be a fixed contraction with the coefficient $\tau \in(0,1)$. Let $\left\{x_{S, T, t}\right\}$ be an sequence defined as follows

$$
x_{S, T, t}=t T x_{t}+(1-t) S x_{S, T, t},
$$

where $t \in(0,1)$. Then $\left\{x_{t}\right\}$ converges strongly as $t \rightarrow 0$ to a fixed point $x^{*}$ of $S$, which is the unique solution in $F(S)$ to the following variational inequality

$$
\left\langle T x^{*}-x^{*}, j\left(x^{*}-p\right)\right\rangle \geq 0, \forall p \in F(S) .
$$

Lemma 4 [2]. In a Banach space E, there holds the inequality

$$
\|x+y\|^{2} \leq\|x\|^{2}+2\langle y, j(x+y)\rangle, \forall x, y \in E,
$$

where $j(x+y) \in J(x+y)$.

\section{Main Results}

Theorem 1. Let E be a reflexive and uniformly convex Banach space which has uniformly Gâteaux differentiable norm and $C$ be a nonempty closed convex subset of $E$ which has normal structure. Let $f: C \rightarrow C$ be a contractive mapping with $k \in[0,1), A$ be a m-accretive operator in $E$ and $S: C \rightarrow C$ be a nonexpansive mapping with $F(S) \cap N(A) \neq \varnothing$. For any $x_{0} \in C$ and $\forall n \geq 0,\left\{x_{n}\right\}$ is generated by

$$
\left\{\begin{array}{l}
y_{n}=\beta_{n}\left(\frac{x_{n}+x_{n+1}}{2}\right)+\left(1-\beta_{n}\right) J_{r_{n}}\left(\frac{x_{n}+x_{n+1}}{2}\right), \\
x_{n+1}=\alpha_{n} f x_{n}+\left(1-\alpha_{n}\right) S y_{n},
\end{array}\right.
$$

where $\left\{\alpha_{n}\right\},\left\{\beta_{n}\right\} \subset(0,1)$ and $\left\{r_{n}\right\} \subset(0,1)$ satisfy the following conditions: 
(i) $\lim _{n \rightarrow \infty} \alpha_{n}=0, \sum_{n=0}^{\infty} \alpha_{n}=\infty,\left|\alpha_{n}-\alpha_{n-1}\right|=o\left(\alpha_{n}\right)$; (ii) $\sum_{n=1}^{\infty}\left|\beta_{n}-\beta_{n-1}\right|<\infty$; (iii) $\lim _{n \rightarrow \infty} r_{n}=r$, $\sum_{n=1}^{\infty}\left|r_{n}-r_{n-1}\right|<\infty$.

Then $\left\{x_{n}\right\}$ and $\left\{y_{n}\right\}$ converge strongly to $q \in F(S) \cap N(A)$, where $q$ is the unique solution of the variational inequality $\left\langle(I-f) q, J_{\varphi}(q-p)\right\rangle \leq 0, \forall p \in F(S) \cap N(A)$.

Proof. The proof is split into eleven steps.

Step 1: Show that $\left\{x_{n}\right\}$ and $\left\{y_{n}\right\}$ are bounded.

Take $p \in F(S) \cap N(A)$, then we have

$$
\begin{aligned}
\left\|y_{n}-p\right\| & \leq \beta_{n}\left\|\frac{x_{n}+x_{n+1}}{2}-p\right\|+\left(1-\beta_{n}\right)\left\|J_{r_{n}}\left(\frac{x_{n}+x_{n+1}}{2}\right)-p\right\| \\
& \leq \beta_{n}\left\|\frac{x_{n}+x_{n+1}}{2}-p\right\|+\left(1-\beta_{n}\right)\left\|\frac{x_{n}+x_{n+1}}{2}-p\right\| \\
& \leq \frac{1}{2}\left\|x_{n}-p\right\|+\frac{1}{2}\left\|x_{n+1}-p\right\|,
\end{aligned}
$$

and then we get

$$
\begin{aligned}
\left\|x_{n+1}-p\right\| & \leq \alpha_{n}\left\|f x_{n}-p\right\|+\left(1-\alpha_{n}\right)\left\|S y_{n}-p\right\| \\
& \leq k \alpha_{n}\left\|x_{n}-p\right\|+\alpha_{n}\|f p-p\|+\left(1-\alpha_{n}\right)\left\|y_{n}-p\right\| \\
& \leq k \alpha_{n}\left\|x_{n}-p\right\|+\alpha_{n}\|f p-p\|+\frac{1-\alpha_{n}}{2}\left\|x_{n}-p\right\|+\frac{1-\alpha_{n}}{2}\left\|x_{n+1}-p\right\| \\
& =\left(k \alpha_{n}+\frac{1-\alpha_{n}}{2}\right)\left\|x_{n}-p\right\|+\alpha_{n}\|f p-p\|+\frac{1-\alpha_{n}}{2}\left\|x_{n+1}-p\right\| .
\end{aligned}
$$

It follows that

$$
\begin{aligned}
\left\|x_{n+1}-p\right\| & \leq \frac{k \alpha_{n}+\frac{1-\alpha_{n}}{2}}{\frac{1+\alpha_{n}}{2}}\left\|x_{n}-p\right\|+\frac{2 \alpha_{n}}{1+\alpha_{n}}\|f p-p\| \\
& =\left[1-\frac{2 \alpha_{n}(1-k)}{1+\alpha_{n}}\right]\left\|x_{n}-p\right\|+\frac{2 \alpha_{n}(1-k)}{1+\alpha_{n}} \frac{\|f p-p\|}{1-k} \\
& \leq \max \left\{\left\|x_{0}-p\right\|, \frac{\|f p-p\|}{1-k}\right\} .
\end{aligned}
$$

Then $\left\{x_{n}\right\}$ and $\left\{y_{n}\right\}$ are bounded. So $\left\{f x_{n}\right\},\left\{f y_{n}\right\},\left\{S x_{n}\right\},\left\{S y_{n}\right\},\left\{J_{r_{n}} x_{n}\right\}$ and $\left\{J_{r_{n}} y_{n}\right\}$ are also bounded.

Step 2: Show that $\lim _{n \rightarrow \infty}\left\|x_{n+1}-x_{n}\right\|=0$.

From (2), we have

$$
\begin{aligned}
\left\|x_{n+1}-x_{n}\right\| & =\left\|\alpha_{n} f x_{n}+\left(1-\alpha_{n}\right) S y_{n}-\alpha_{n-1} f x_{n-1}-\left(1-\alpha_{n-1}\right) S y_{n-1}\right\| \\
& \leq \alpha_{n}\left\|f x_{n}-f x_{n-1}\right\|+\left|\alpha_{n}-\alpha_{n-1}\right| \cdot\left\|f x_{n-1}-S y_{n-1}\right\|+\left(1-\alpha_{n}\right)\left\|S y_{n}-S y_{n-1}\right\| \\
& \leq k \alpha_{n}\left\|x_{n}-x_{n-1}\right\|+\left|\alpha_{n}-\alpha_{n-1}\right| \cdot\left\|f x_{n-1}-S y_{n-1}\right\|+\left(1-\alpha_{n}\right)\left\|y_{n}-y_{n-1}\right\| .
\end{aligned}
$$

From (2), we have

$$
\begin{aligned}
\left\|y_{n}-y_{n-1}\right\| & =\| \beta_{n}\left(\frac{x_{n}+x_{n+1}}{2}\right)+\left(1-\beta_{n}\right) J_{r_{n}}\left(\frac{x_{n}+x_{n+1}}{2}\right)-\beta_{n-1}\left(\frac{x_{n-1}+x_{n}}{2}\right) \\
& -\left(1-\beta_{n-1}\right) J_{r_{n-1}}\left(\frac{x_{n-1}+x_{n}}{2}\right) \| \\
& \leq \beta_{n}\left\|\frac{x_{n+1}-x_{n-1}}{2}\right\|+\left|\beta_{n}-\beta_{n-1}\right| \cdot\left\|\frac{x_{n-1}+x_{n}}{2}-J_{r_{n-1}}\left(\frac{x_{n-1}+x_{n}}{2}\right)\right\| \\
& +\left(1-\beta_{n}\right)\left\|J_{r_{n}}\left(\frac{x_{n}+x_{n+1}}{2}\right)-J_{r_{n-1}}\left(\frac{x_{n-1}+x_{n}}{2}\right)\right\| .
\end{aligned}
$$


From Lemma 1, we have

$$
\begin{aligned}
& \left\|J_{r_{n}}\left(\frac{x_{n}+x_{n+1}}{2}\right)-J_{r_{n-1}}\left(\frac{x_{n-1}+x_{n}}{2}\right)\right\| \\
& =\left\|J_{r_{n-1}}\left(\frac{r_{n-1}}{r_{n}}\left(\frac{x_{n}+x_{n+1}}{2}\right)+\left(1-\frac{r_{n-1}}{r_{n}}\right) J_{r_{n}}\left(\frac{x_{n}+x_{n+1}}{2}\right)\right)-J_{r_{n-1}}\left(\frac{x_{n-1}+x_{n}}{2}\right)\right\| \\
& \leq\left\|\frac{r_{n-1}}{r_{n}}\left(\frac{x_{n}+x_{n+1}}{2}\right)+\left(1-\frac{r_{n-1}}{r_{n}}\right) J_{r_{n}}\left(\frac{x_{n}+x_{n+1}}{2}\right)-\frac{x_{n-1}+x_{n}}{2}\right\| \\
& =\left\|\frac{r_{n-1}}{r_{n}}\left(\frac{x_{n+1}-x_{n-1}}{2}\right)+\left(1-\frac{r_{n-1}}{r_{n}}\right)\left(J_{r_{n}}\left(\frac{x_{n}+x_{n+1}}{2}\right)-\frac{x_{n-1}+x_{n}}{2}\right)\right\| \\
& \leq \frac{r_{n-1}}{r_{n}}\left\|\frac{x_{n+1}-x_{n-1}}{2}\right\|+\left|1-\frac{r_{n-1}}{r_{n}}\right| \cdot\left\|J_{r_{n}}\left(\frac{x_{n}+x_{n+1}}{2}\right)-\frac{x_{n}+x_{n+1}}{2}\right\|+\left|1-\frac{r_{n-1}}{r_{n}}\right| \cdot\left\|\frac{x_{n+1}-x_{n-1}}{2}\right\| \\
& =\left\|\frac{x_{n+1}-x_{n-1}}{2}\right\|+\left|1-\frac{r_{n-1}}{r_{n}}\right| \cdot\left\|J_{r_{n}}\left(\frac{x_{n}+x_{n+1}}{2}\right)-\frac{x_{n}+x_{n+1}}{2}\right\| .
\end{aligned}
$$

Put (4) and (5) into (3), we get

$$
\begin{aligned}
\left\|x_{n+1}-x_{n}\right\| & \leq k \alpha_{n}\left\|x_{n}-x_{n-1}\right\|+\left|\alpha_{n}-\alpha_{n-1}\right| \cdot\left\|f x_{n-1}-S y_{n-1}\right\|+\left(1-\alpha_{n}\right)\left\|\frac{x_{n+1}-x_{n-1}}{2}\right\| \\
& +\left(1-\alpha_{n}\right)\left|\beta_{n}-\beta_{n-1}\right| \cdot\left\|\frac{x_{n-1}+x_{n}}{2}-J_{r_{n-1}}\left(\frac{x_{n-1}+x_{n}}{2}\right)\right\| \\
& +\left(1-\alpha_{n}\right)\left(1-\beta_{n}\right)\left|1-\frac{r_{n-1}}{r_{n}}\right| \cdot\left\|\frac{x_{n}+x_{n+1}}{2}-J_{r_{n}}\left(\frac{x_{n}+x_{n+1}}{2}\right)\right\| \\
& \leq k \alpha_{n}\left\|x_{n}-x_{n-1}\right\|+\frac{1-\alpha_{n}}{2}\left\|x_{n+1}-x_{n}\right\|+\frac{1-\alpha_{n}}{2}\left\|x_{n}-x_{n-1}\right\|+\left|\alpha_{n}-\alpha_{n-1}\right| M_{1} \\
& +\left[\left(1-\alpha_{n}\right)\left|\beta_{n}-\beta_{n-1}\right|+\left(1-\alpha_{n}\right)\left(1-\beta_{n}\right)\left|1-\frac{r_{n-1}}{r_{n}}\right|\right] M_{2} \\
& =\left(k \alpha_{n}+\frac{1-\alpha_{n}}{2}\right)\left\|x_{n}-x_{n-1}\right\|+\frac{1-\alpha_{n}}{2}\left\|x_{n}-x_{n-1}\right\|+\left|\alpha_{n}-\alpha_{n-1}\right| M_{1} \\
& +\left[\left(1-\alpha_{n}\right)\left|\beta_{n}-\beta_{n-1}\right|+\left(1-\alpha_{n}\right)\left(1-\beta_{n}\right)\left|1-\frac{r_{n-1}}{r_{n}}\right|\right] M_{2} .
\end{aligned}
$$

It follows that

$$
\begin{aligned}
\left\|x_{n+1}-x_{n}\right\| & \leq \frac{k \alpha_{n}+\frac{1-\alpha_{n}}{2}}{\frac{1+\alpha_{n}}{2}}\left\|x_{n}-x_{n-1}\right\|+\frac{2\left|\alpha_{n}-\alpha_{n-1}\right|}{1+\alpha_{n}} M_{1} \\
& +\frac{2\left(1-\alpha_{n}\right)\left|\beta_{n}-\beta_{n-1}\right|+2\left(1-\alpha_{n}\right)\left(1-\beta_{n}\right)\left|1-\frac{r_{n-1}}{r_{n}}\right|}{1+\alpha_{n}} M_{2} \\
& =\left[1-\frac{2 \alpha_{n}(1-k)}{1+\alpha_{n}}\right]|| x_{n}-x_{n-1} \|+\frac{2\left|\alpha_{n}-\alpha_{n-1}\right|}{1+\alpha_{n}} M_{1} \\
& +\frac{2\left(1-\alpha_{n}\right)\left|\beta_{n}-\beta_{n-1}\right|+2\left(1-\alpha_{n}\right)\left(1-\beta_{n}\right)\left|1-\frac{r_{n-1}}{r_{n}}\right|}{1+\alpha_{n}} M_{2}
\end{aligned}
$$

where $M_{1}=\max \left\|f x_{n-1}-S y_{n-1}\right\|$ and $M_{2}=\max \left\|\frac{x_{n}+x_{n+1}}{2}-J_{r_{n}}\left(\frac{x_{n}+x_{n+1}}{2}\right)\right\|$.

Take $t_{n}=\frac{2 \alpha_{n}(1-k)}{1+\alpha_{n}}$, then $t_{n}>\alpha_{n}(1-k)$. From $\sum_{n=0}^{\infty} \alpha_{n}=\infty$, so $\sum_{n=0}^{\infty} t_{n}=\infty$.

Take $b_{n}=\frac{2\left|\alpha_{n}-\alpha_{n-1}\right|}{1+\alpha_{n}} M_{1}$, then $\frac{b_{n}}{t_{n}}=\frac{\left|\alpha_{n}-\alpha_{n-1}\right| M_{1}}{\alpha_{n}(1-k)}$. From $\left|\alpha_{n}-\alpha_{n-1}\right|=o\left(\alpha_{n}\right)$, so $b_{n}=o\left(t_{n}\right)$.

Take $c_{n}=\frac{2\left(1-\alpha_{n}\right)\left|\beta_{n}-\beta_{n-1}\right|+2\left(1-\alpha_{n}\right)\left(1-\beta_{n}\right)\left|1-\frac{r_{n-1}}{r_{n}}\right|}{1+\alpha_{n}} M_{2} . \quad$ From $\lim _{n \rightarrow \infty} r_{n}=r$, then $c_{n}<$ $2 M_{2}\left(\left|\beta_{n}-\beta_{n-1}\right|+\frac{\left|r_{n}-r_{n-1}\right|}{r-\varepsilon}\right)(\forall \varepsilon>0)$. From $\sum_{n=1}^{\infty}\left|\beta_{n}-\beta_{n-1}\right|<\infty$ and $\sum_{n=1}^{\infty}\left|r_{n}-r_{n-1}\right|<\infty$, so $\sum_{n=1}^{\infty} c_{n}<$ $\infty$.

From Lemma 2, we get $\lim _{n \rightarrow \infty}\left\|x_{n+1}-x_{n}\right\|=0$.

Step 3: Show that $\lim _{n \rightarrow \infty}\left\|x_{n}-J_{r_{n}} x_{n}\right\|=0$. 
Because $\|\cdot\|^{2}$ is convex function and (1), so we have

$$
\begin{aligned}
\left\|x_{n+1}-p\right\|^{2} \leq & \alpha_{n}\left\|f x_{n}-p\right\|^{2}+\left(1-\alpha_{n}\right)\left\|S y_{n}-p\right\|^{2} \\
\leq & \alpha_{n}\left\|f x_{n}-p\right\|^{2}+\left(1-\alpha_{n}\right)\left\|y_{n}-p\right\|^{2} \\
\leq & \alpha_{n}\left\|f x_{n}-p\right\|^{2}+\left(1-\alpha_{n}\right)\left[\beta_{n}\left\|\frac{x_{n}+x_{n+1}}{2}-p\right\|^{2}+\left(1-\beta_{n}\right)\left\|J_{r_{n}}\left(\frac{x_{n}+x_{n+1}}{2}\right)-p\right\|^{2}\right. \\
& \left.-\beta_{n}\left(1-\beta_{n}\right) g\left(\left\|\frac{x_{n}+x_{n+1}}{2}-J_{r_{n}}\left(\frac{x_{n}+x_{n+1}}{2}\right)\right\|\right)\right] \\
\leq & \alpha_{n}\left\|f x_{n}-p\right\|^{2}+\left(1-\alpha_{n}\right)\left\|\frac{x_{n}+x_{n+1}}{2}-p\right\|^{2} \\
& -\left(1-\alpha_{n}\right) \beta_{n}\left(1-\beta_{n}\right) g\left(\left\|\frac{x_{n}+x_{n+1}}{2}-J_{r_{n}}\left(\frac{x_{n}+x_{n+1}}{2}\right)\right\|\right) \\
\leq & \alpha_{n}\left\|f x_{n}-p\right\|^{2}+\frac{1-\alpha_{n}}{2}\left\|x_{n}-p\right\|^{2}+\frac{1-\alpha_{n}}{2}\left\|x_{n+1}-p\right\|^{2} \\
& -\left(1-\alpha_{n}\right) \beta_{n}\left(1-\beta_{n}\right) g\left(\left\|\frac{x_{n}+x_{n+1}}{2}-J_{r_{n}}\left(\frac{x_{n}+x_{n+1}}{2}\right)\right\|\right) .
\end{aligned}
$$

It follows that

$$
\begin{aligned}
\left\|x_{n+1}-p\right\|^{2} & \leq \frac{1-\alpha_{n}}{1+\alpha_{n}}\left\|x_{n}-p\right\|^{2}+\frac{2 \alpha_{n}}{1+\alpha_{n}}\left\|f x_{n}-p\right\|^{2} \\
& -\frac{2\left(1-\alpha_{n}\right) \beta_{n}\left(1-\beta_{n}\right)}{1+\alpha_{n}} g\left(\left\|\frac{x_{n}+x_{n+1}}{2}-J_{r_{n}}\left(\frac{x_{n}+x_{n+1}}{2}\right)\right\|\right) \\
& \leq\left\|x_{n}-p\right\|^{2}+\frac{2 \alpha_{n}}{1+\alpha_{n}}\left\|f x_{n}-p\right\|^{2} \\
& -\frac{2\left(1-\alpha_{n}\right) \beta_{n}\left(1-\beta_{n}\right)}{1+\alpha_{n}} g\left(\left\|\frac{x_{n}+x_{n+1}}{2}-J_{r_{n}}\left(\frac{x_{n}+x_{n+1}}{2}\right)\right\|\right) .
\end{aligned}
$$

Then we have

$$
\begin{aligned}
& \frac{2\left(1-\alpha_{n}\right) \beta_{n}\left(1-\beta_{n}\right)}{1+\alpha_{n}} g\left(\left\|\frac{x_{n}+x_{n+1}}{2}-J_{r_{n}}\left(\frac{x_{n}+x_{n+1}}{2}\right)\right\|\right)-\frac{2 \alpha_{n}}{1+\alpha_{n}}\left\|f x_{n}-p\right\|^{2} \\
\leq & \left\|x_{n}-p\right\|^{2}-\left\|x_{n+1}-p\right\|^{2} .
\end{aligned}
$$

If $\frac{2\left(1-\alpha_{n}\right) \beta_{n}\left(1-\beta_{n}\right)}{1+\alpha_{n}} g\left(\left\|\frac{x_{n}+x_{n+1}}{2}-J_{r_{n}}\left(\frac{x_{n}+x_{n+1}}{2}\right)\right\|\right) \leq \frac{2 \alpha_{n}}{1+\alpha_{n}}\left\|f x_{n}-p\right\|^{2}$, so from $\lim _{n \rightarrow \infty} \alpha_{n}=0$ and the boundedness of $\left\{f x_{n}\right\}$, we get $\lim _{n \rightarrow \infty} g\left(\left\|\frac{x_{n}+x_{n+1}}{2}-J_{r_{n}}\left(\frac{x_{n}+x_{n+1}}{2}\right)\right\|\right)=0$.

$$
\begin{aligned}
& \text { If } \frac{2\left(1-\alpha_{n}\right) \beta_{n}\left(1-\beta_{n}\right)}{1+\alpha_{n}} g\left(\left\|\frac{x_{n}+x_{n+1}}{2}-J_{r_{n}}\left(\frac{x_{n}+x_{n+1}}{2}\right)\right\|\right)>\frac{2 \alpha_{n}}{1+\alpha_{n}}\left\|f x_{n}-p\right\|^{2} \text {, so } \\
& \sum_{n=0}^{N}\left[\frac{2\left(1-\alpha_{n}\right) \beta_{n}\left(1-\beta_{n}\right)}{1+\alpha_{n}} g\left(\left\|\frac{x_{n}+x_{n+1}}{2}-J_{r_{n}}\left(\frac{x_{n}+x_{n+1}}{2}\right)\right\|\right)-\frac{2 \alpha_{n}}{1+\alpha_{n}}\left\|f x_{n}-p\right\|^{2}\right] \\
& \leq\left\|x_{0}-p\right\|^{2}-\left\|x_{N+1}-p\right\|^{2} \leq\left\|x_{0}-p\right\|^{2} .
\end{aligned}
$$

Then $\sum_{n=0}^{\infty}\left[\frac{2\left(1-\alpha_{n}\right) \beta_{n}\left(1-\beta_{n}\right)}{1+\alpha_{n}} g\left(\left\|\frac{x_{n}+x_{n+1}}{2}-J_{r_{n}}\left(\frac{x_{n}+x_{n+1}}{2}\right)\right\|\right)-\frac{2 \alpha_{n}}{1+\alpha_{n}}\left\|f x_{n}-p\right\|^{2}\right]<\infty$.

So we get

$$
\lim _{n \rightarrow \infty}\left[\frac{2\left(1-\alpha_{n}\right) \beta_{n}\left(1-\beta_{n}\right)}{1+\alpha_{n}} g\left(\left\|\frac{x_{n}+x_{n+1}}{2}-J_{r_{n}}\left(\frac{x_{n}+x_{n+1}}{2}\right)\right\|\right)-\frac{2 \alpha_{n}}{1+\alpha_{n}}\left\|f x_{n}-p\right\|^{2}\right]=0,
$$

and then $\lim _{n \rightarrow \infty} g\left(\left\|\frac{x_{n}+x_{n+1}}{2}-J_{r_{n}}\left(\frac{x_{n}+x_{n+1}}{2}\right)\right\|\right)=0$.

From the property of $g$, so we get $\lim _{n \rightarrow \infty}\left\|\frac{x_{n}+x_{n+1}}{2}-J_{r_{n}}\left(\frac{x_{n}+x_{n+1}}{2}\right)\right\|=0$.

We also have

$$
\begin{gathered}
\left\|x_{n}-J_{r_{n}} x_{n}\right\| \leq\left\|x_{n}-\frac{x_{n}+x_{n+1}}{2}\right\|+\left\|\frac{x_{n}+x_{n+1}}{2}-J_{r_{n}}\left(\frac{x_{n}+x_{n+1}}{2}\right)\right\|+\left\|J_{r_{n}}\left(\frac{x_{n}+x_{n+1}}{2}\right)-J_{r_{n}} x_{n}\right\| \\
\leq\left\|x_{n+1}-x_{n}\right\|+\left\|\frac{x_{n}+x_{n+1}}{2}-J_{r_{n}}\left(\frac{x_{n}+x_{n+1}}{2}\right)\right\| .
\end{gathered}
$$


Then from step 2, we get $\lim _{n \rightarrow \infty}\left\|x_{n}-J_{r_{n}} x_{n}\right\|=0$.

Step 4: Show that $\lim _{n \rightarrow \infty}\left\|y_{n}-S y_{n}\right\|=0$.

$$
\begin{aligned}
\left\|y_{n}-S y_{n}\right\| & \leq \beta_{n}\left\|\frac{x_{n}+x_{n+1}}{2}-S y_{n}\right\|+\left(1-\beta_{n}\right)\left\|J_{r_{n}}\left(\frac{x_{n}+x_{n+1}}{2}\right)-S y_{n}\right\| \\
& \leq\left\|\frac{x_{n}+x_{n+1}}{2}-S y_{n}\right\|+\left(1-\beta_{n}\right)\left\|J_{r_{n}}\left(\frac{x_{n}+x_{n+1}}{2}\right)-\frac{x_{n}+x_{n+1}}{2}\right\| \\
& \leq\left\|\frac{x_{n}+x_{n+1}}{2}-x_{n+1}\right\|+\left\|x_{n+1}-S y_{n}\right\|+\left(1-\beta_{n}\right)\left\|J_{r_{n}}\left(\frac{x_{n}+x_{n+1}}{2}\right)-\frac{x_{n}+x_{n+1}}{2}\right\| \\
& =\frac{1}{2}\left\|x_{n}-x_{n+1}\right\|+\alpha_{n}\left\|f x_{n}-S y_{n}\right\|+\left(1-\beta_{n}\right)\left\|J_{r_{n}}\left(\frac{x_{n}+x_{n+1}}{2}\right)-\frac{x_{n}+x_{n+1}}{2}\right\| .
\end{aligned}
$$

From steps 2 and $3, \lim _{n \rightarrow \infty} \alpha_{n}=0$ and the boundedness of $\left\{f x_{n}\right\}$ and $\left\{S y_{n}\right\}$, we get $\lim _{n \rightarrow \infty}\left\|y_{n}-S y_{n}\right\|=0$.

Step 5: Show that $\lim _{n \rightarrow \infty}\left\|x_{n}-y_{n}\right\|=0$.

$$
\begin{aligned}
\left\|x_{n}-y_{n}\right\| & \leq\left\|x_{n}-x_{n+1}\right\|+\left\|x_{n+1}-S y_{n}\right\|+\left\|S y_{n}-y_{n}\right\| \\
& =\left\|x_{n}-x_{n+1}\right\|+\alpha_{n}\left\|f x_{n}-S y_{n}\right\|+\left\|S y_{n}-y_{n}\right\| .
\end{aligned}
$$

From steps 2 and 4 , we get $\lim _{n \rightarrow \infty}\left\|x_{n}-y_{n}\right\|=0$.

Step 6: Show that $\lim _{n \rightarrow \infty}\left\|y_{n}-J_{r_{n}} y_{n}\right\|=0$.

$$
\begin{aligned}
\left\|y_{n}-J_{r_{n}} y_{n}\right\| & \leq\left\|y_{n}-x_{n}\right\|+\left\|x_{n}-J_{r_{n}} x_{n}\right\|+\left\|J_{r_{n}} x_{n}-J_{r_{n}} y_{n}\right\| \\
& \leq 2\left\|y_{n}-x_{n}\right\|+\left\|x_{n}-J_{r_{n}} x_{n}\right\| .
\end{aligned}
$$

From steps 3 and 5, we get $\lim _{n \rightarrow \infty}\left\|y_{n}-J_{r_{n}} y_{n}\right\|=0$.

Step 7: Show that $\lim _{n \rightarrow \infty}\left\|x_{n}-S x_{n}\right\|=0$.

$$
\begin{aligned}
\left\|x_{n}-S x_{n}\right\| & \leq\left\|x_{n}-y_{n}\right\|+\left\|y_{n}-S y_{n}\right\|+\left\|S y_{n}-S x_{n}\right\| \\
& \leq 2\left\|x_{n}-y_{n}\right\|+\left\|y_{n}-S y_{n}\right\| .
\end{aligned}
$$

From steps 4 and 5, we get $\lim _{n \rightarrow \infty}\left\|x_{n}-S x_{n}\right\|=0$.

Step 8: Show that $\lim _{n \rightarrow \infty}\left\|y_{n}-J_{r} y_{n}\right\|=0$.

$$
\begin{aligned}
\left\|y_{n}-J_{r} y_{n}\right\| & \leq\left\|y_{n}-J_{r_{n}} y_{n}\right\|+\left\|J_{r_{n}} y_{n}-J_{r} y_{n}\right\| \\
& =\left\|y_{n}-J_{r_{n}} y_{n}\right\|+\left\|J_{r}\left(\frac{r}{r_{n}} y_{n}+\left(1-\frac{r}{r_{n}}\right) J_{r_{n}} y_{n}\right)-J_{r} y_{n}\right\| \\
& \leq\left\|y_{n}-J_{r_{n}} y_{n}\right\|+\left|1-\frac{r}{r_{n}}\right| \cdot\left\|J_{r_{n}} y_{n}-y_{n}\right\| .
\end{aligned}
$$

From step 6 and $\lim _{n \rightarrow \infty} r_{n}=r$, we get $\lim _{n \rightarrow \infty}\left\|y_{n}-J_{r} y_{n}\right\|=0$.

Step 9: Show that $\lim _{n \rightarrow \infty}\left\|x_{n}-J_{r} x_{n}\right\|=0$.

$$
\begin{aligned}
\left\|x_{n}-J_{r} x_{n}\right\| & \leq\left\|x_{n}-y_{n}\right\|+\left\|y_{n}-J_{r} y_{n}\right\|+\left\|J_{r} y_{n}-J_{r} x_{n}\right\| \\
& \leq 2\left\|x_{n}-y_{n}\right\|+\left\|y_{n}-J_{r} y_{n}\right\| .
\end{aligned}
$$

From steps 5 and 8 , we get $\lim _{n \rightarrow \infty}\left\|x_{n}-J_{r} x_{n}\right\|=0$.

Step 10: Show that $\limsup _{n \rightarrow \infty}\left\langle q-f q, J\left(q-x_{n}\right)\right\rangle=0$. 
Let $\left\{x_{t}\right\}$ be defined by $x_{t}=t f x_{t}+(1-t) S x_{t}$. From Lemma 3, we have that $\left\{x_{t}\right\}$ converges strongly to $q \in P_{F(S) \cap N(A)} f q$, which is also the unique solution of the variational inequality $\langle q-f q, J(q-p)\rangle \leq 0, \forall p \in F(S) \cap N(A)$.

We have

$$
\begin{aligned}
\left\|x_{t}-x_{n}\right\|^{2} & =(1-t)\left\langle S x_{t}-S x_{n}+S x_{n}-x_{n}, J\left(x_{t}-x_{n}\right)\right\rangle+t\left\langle f x_{t}-x_{t}+x_{t}-x_{n}, J\left(x_{t}-x_{n}\right)\right\rangle \\
& \leq(1-t)\left\|x_{t}-x_{n}\right\|^{2}+(1-t)\left\|S x_{n}-x_{n}\right\| \cdot\left\|x_{t}-x_{n}\right\|+t\left\|x_{t}-x_{n}\right\|^{2}+t\left\langle f x_{t}-x_{t}, J\left(x_{t}-x_{n}\right)\right\rangle \\
& =\left\|x_{t}-x_{n}\right\|^{2}+(1-t)\left\|S x_{n}-x_{n}\right\| \cdot\left\|x_{t}-x_{n}\right\|+t\left\|x_{t}-x_{n}\right\|^{2}+t\left\langle f x_{t}-x_{t}, J\left(x_{t}-x_{n}\right)\right\rangle .
\end{aligned}
$$

It follows that $\left\langle f x_{t}-x_{t}, J\left(x_{t}-x_{n}\right)\right\rangle \leq \frac{1-t}{t}\left\|S x_{n}-x_{n}\right\| \cdot\left\|x_{t}-x_{n}\right\|$. From step 7, we get $\limsup \left\langle q-f q, J\left(q-x_{n}\right)\right\rangle=0$.

Step 11: Show that $\lim _{n \rightarrow \infty}\left\|x_{n}-q\right\|=0$.

From Lemma 4, we have

$$
\begin{aligned}
\left\|x_{n+1}-q\right\|^{2} & \leq\left(1-\alpha_{n}\right)^{2}\left\|S y_{n}-q\right\|^{2}+2 \alpha_{n}\left\langle f x_{n}-q, J\left(x_{n+1}-q\right)\right\rangle \\
& \leq\left(1-\alpha_{n}\right)^{2}\left\|y_{n}-q\right\|^{2}+2 k \alpha_{n}\left\|x_{n}-q\right\| \cdot\left\|x_{n+1}-q\right\|+2 \alpha_{n}\left\langle f q-q, J\left(x_{n+1}-q\right)\right\rangle \\
& \leq\left(1-\alpha_{n}\right)^{2}\left(\frac{1}{2}\left\|x_{n}-q\right\|+\frac{1}{2}\left\|x_{n+1}-q\right\|\right)^{2}+2 k \alpha_{n}\left\|x_{n}-q\right\| \cdot\left\|x_{n+1}-q\right\| \\
& +2 \alpha_{n}\left\langle f q-q, J\left(x_{n+1}-q\right)\right\rangle \\
& =\left(\frac{1-\alpha_{n}}{2}\right)^{2}\left\|x_{n}-q\right\|^{2}+\left(\frac{1-\alpha_{n}}{2}\right)^{2}\left\|x_{n+1}-q\right\|^{2}+\frac{\left(1-\alpha_{n}\right)^{2}}{2}\left\|x_{n}-q\right\| \cdot\left\|x_{n+1}-q\right\| \\
& +2 k \alpha_{n}\left\|x_{n}-q\right\| \cdot\left\|x_{n+1}-q\right\|+2 \alpha_{n}\left\langle f q-q, J\left(x_{n+1}-q\right)\right\rangle \\
& =\left(\frac{1-\alpha_{n}}{2}\right)^{2}\left\|x_{n}-q\right\|^{2}+\left(\frac{1-\alpha_{n}}{2}\right)^{2}\left\|x_{n+1}-q\right\|^{2} \\
& +\left[\frac{\left(1-\alpha_{n}\right)^{2}}{2}+2 k \alpha_{n}\right]\left\|x_{n}-q\right\| \cdot\left\|x_{n+1}-q\right\|+2 \alpha_{n}\left\langle f q-q, J\left(x_{n+1}-q\right)\right\rangle \\
& \leq\left(\frac{1-\alpha_{n}}{2}\right)^{2}\left\|x_{n}-q\right\|^{2}+\left(\frac{1-\alpha_{n}}{2}\right)^{2}\left\|x_{n+1}-q\right\|^{2} \\
& +\left[\frac{\left(1-\alpha_{n}\right)^{2}}{4}+k \alpha_{n}\right]\left(\left\|x_{n}-q\right\|^{2}+\left\|x_{n+1}-q\right\|^{2}\right)+2 \alpha_{n}\left\langle f q-q, J\left(x_{n+1}-q\right)\right\rangle .
\end{aligned}
$$

It follows that

$$
\begin{aligned}
\left\|x_{n+1}-q\right\|^{2} & \leq \frac{\left(\frac{1-\alpha_{n}}{2}\right)^{2}+\frac{\left(1-\alpha_{n}\right)^{2}}{4}+k \alpha_{n}}{1-\left[\left(\frac{1-\alpha_{n}}{2}\right)^{2}+\frac{\left(1-\alpha_{n}\right)^{2}}{4}+k \alpha_{n}\right]}\left\|x_{n}-q\right\|^{2} \\
& +\frac{2 \alpha_{n}}{1-\left[\left(\frac{1-\alpha_{n}}{2}\right)^{2}+\frac{\left(1-\alpha_{n}\right)^{2}}{4}+k \alpha_{n}\right]}\left\langle f q-q, J\left(x_{n+1}-q\right)\right\rangle \\
& =\left[1-\frac{1-2\left(\frac{\left(1-\alpha_{n}\right)^{2}}{2}+k \alpha_{n}\right)}{1-\left(\frac{\left(1-\alpha_{n}\right)^{2}}{2}+k \alpha_{n}\right)}\right]\left\|x_{n}-q\right\|^{2}+\frac{2 \alpha_{n}}{1-\left[\frac{\left(1-\alpha_{n}\right)^{2}}{2}+k \alpha_{n}\right]}\left\langle f q-q, J\left(x_{n+1}-q\right)\right\rangle .
\end{aligned}
$$

Take $t_{n}=\frac{1-2\left(\frac{\left(1-\alpha_{n}\right)^{2}}{2}+k \alpha_{n}\right)}{1-\left(\frac{\left(1-\alpha_{n}\right)^{2}}{2}+k \alpha_{n}\right)}$. From $\lim _{n \rightarrow \infty} \alpha_{n}=0$, we have

$$
t_{n} \geq 1-2\left(\frac{\left(1-\alpha_{n}\right)^{2}}{2}+k \alpha_{n}\right)=\alpha_{n}\left(2-2 k-\alpha_{n}\right) \geq \alpha_{n}(2-2 k-\varepsilon)(\forall \varepsilon>0) .
$$

From $\sum_{n=0}^{\infty} \alpha_{n}=\infty$, we get $\sum_{n=0}^{\infty} t_{n}=\infty$ 
Take $b_{n}=\frac{2 \alpha_{n}}{1-\left[\frac{\left(1-\alpha_{n}\right)^{2}}{2}+k \alpha_{n}\right]}\left\langle f q-q, J\left(x_{n+1}-q\right)\right\rangle$, then we have

$$
\frac{b_{n}}{t_{n}}=\frac{2 \alpha_{n}\left\langle f q-q_{,} J\left(x_{n+1}-q\right)\right\rangle}{1-2\left[\frac{\left(1-\alpha_{n}\right)^{2}}{2}+k \alpha_{n}\right]}=\frac{2\left\langle f q-q, J\left(x_{n+1}-q\right)\right\rangle}{2-2 k-\alpha_{n}} .
$$

From $\lim _{n \rightarrow \infty} \alpha_{n}=0$ and step 10, we get $b_{n}=o\left(t_{n}\right)$.

Take $c_{n}=0$, then we get $\sum_{n=0}^{\infty} c_{n}<\infty$.

From Lemma 2, we get $\lim _{n \rightarrow \infty}\left\|x_{n}-q\right\|=0$. This completes the proof.

The results of Theorem 1 improve the related results in $[13,14,16,17]$. For example, this paper uses the viscosity implicit midpoint rule to find common points of the fixed point set of a nonexpansive mapping and the zero point set of an accretive operator and the results improve the related results in $[13,14]$; If $\beta_{n}=0$, the results of Theorem 1 can obtain the related results in $[16,17]$.

Corollary 1. Let E be a reflexive and uniformly convex Banach space which has uniformly Gâteaux differentiable norm and $C$ be a nonempty closed convex subset of $E$ which has normal structure. Let $f: C \rightarrow C$ be a contractive mapping with $k \in[0,1), A$ be a m-accretive operator in $E$ and $S: C \rightarrow C$ be a nonexpansive mapping with $F(S) \cap N(A) \neq \varnothing$. For any $x_{0} \in C$ and $\forall n \geq 0,\left\{x_{n}\right\}$ is generated by

$$
\left\{\begin{array}{l}
y_{n}=\beta_{n}\left(\frac{x_{n}+x_{n+1}}{2}\right)+\left(1-\beta_{n}\right) J_{r_{n}}\left(\frac{x_{n}+x_{n+1}}{2}\right)+e_{n} \\
x_{n+1}=\alpha_{n} f x_{n}+\left(1-\alpha_{n}\right) S y_{n}
\end{array}\right.
$$

where $\left\{\alpha_{n}\right\},\left\{\beta_{n}\right\} \subset(0,1),\left\{e_{n}\right\} \subset E$ and $\left\{r_{n}\right\} \subset(0,1)$ satisfy the following conditions:

(i) $\lim _{n \rightarrow \infty} \alpha_{n}=0, \sum_{n=0}^{\infty} \alpha_{n}=\infty,\left|\alpha_{n}-\alpha_{n-1}\right|=o\left(\alpha_{n}\right)$;

(ii) $\sum_{n=1}^{\infty}\left|\beta_{n}-\beta_{n-1}\right|<\infty$;

(iii) $\lim _{n \rightarrow \infty} r_{n}=r, \sum_{n=1}^{\infty}\left|r_{n}-r_{n-1}\right|<\infty$;

(iv) $\left\|e_{n}\right\|=o\left(\alpha_{n}\right)$.

Then $\left\{x_{n}\right\}$ and $\left\{y_{n}\right\}$ converge strongly to $q \in F(S) \cap N(A)$, where $q$ is the unique solution of the variational inequality $\left\langle(I-f) q, J_{\varphi}(q-p)\right\rangle \leq 0, \forall p \in F(S) \cap N(A)$.

Proof. Assume

$$
\left\{\begin{array}{l}
w_{n}=\beta_{n}\left(\frac{z_{n}+z_{n+1}}{2}\right)+\left(1-\beta_{n}\right) J_{r_{n}}\left(\frac{z_{n}+z_{n+1}}{2}\right) \\
z_{n+1}=\alpha_{n} f z_{n}+\left(1-\alpha_{n}\right) S w_{n} .
\end{array}\right.
$$

Then we have

$$
\begin{aligned}
\left\|x_{n+1}-z_{n+1}\right\| & \leq k \alpha_{n}\left\|x_{n}-z_{n}\right\|+\left(1-\alpha_{n}\right)\left\|y_{n}-w_{n}\right\| \\
& \leq k \alpha_{n}\left\|x_{n}-z_{n}\right\|+\left(1-\alpha_{n}\right)\left(\left\|\frac{x_{n}+x_{n+1}}{2}-\frac{z_{n}+z_{n+1}}{2}\right\|+\left\|e_{n}\right\|\right) \\
& \leq\left(k \alpha_{n}+\frac{1-\alpha_{n}}{2}\right)\left\|x_{n}-z_{n}\right\|+\frac{1-\alpha_{n}}{2}\left\|x_{n+1}-z_{n+1}\right\|+\left(1-\alpha_{n}\right)\left\|e_{n}\right\| .
\end{aligned}
$$

It follows that

$$
\begin{aligned}
\left\|x_{n+1}-z_{n+1}\right\| & \leq \frac{k \alpha_{n}+\frac{1-\alpha_{n}}{2}}{\frac{1+\alpha_{n}}{2}}\left\|x_{n}-z_{n}\right\|+\frac{2\left(1-\alpha_{n}\right)}{1+\alpha_{n}}\left\|e_{n}\right\| \\
& =\left[1-\frac{2 \alpha_{n}(1-k)}{1+\alpha_{n}}\right]\left\|x_{n}-z_{n}\right\|+\frac{2\left(1-\alpha_{n}\right)}{1+\alpha_{n}}\left\|e_{n}\right\|
\end{aligned}
$$


Take $t_{n}=\frac{2 \alpha_{n}(1-k)}{1+\alpha_{n}}$, then $t_{n} \geq \alpha_{n}(1-k)$. From $\sum_{n=0}^{\infty} \alpha_{n}=\infty$, we get $\sum_{n=0}^{\infty} t_{n}=\infty$.

Take $b_{n}=\frac{2\left(1-\alpha_{n}\right)}{1+\alpha_{n}}\left\|e_{n}\right\|$, then $\frac{b_{n}}{t_{n}}=\frac{\left(1-\alpha_{n}\right)\left\|e_{n}\right\|}{\alpha_{n}(1-k)}$. From $\left\|e_{n}\right\|=o\left(\alpha_{n}\right)$, we get $b_{n}=o\left(t_{n}\right)$.

Take $c_{n}=0$, then we get $\sum_{n=0}^{\infty} c_{n}<\infty$.

From Lemma 2, we get $\lim _{n \rightarrow \infty}\left\|x_{n}-z_{n}\right\|=0$. From Theorem 1, we have $\left\{z_{n}\right\}$ and $\left\{w_{n}\right\}$ converge strongly to $q \in F(S) \cap N(A)$, where $q$ is the unique solution of the variational inequality $\left\langle(I-f) q, J_{\varphi}(q-p)\right\rangle \leq 0, \forall p \in F(S) \cap N(A)$. So $\left\{x_{n}\right\}$ and $\left\{y_{n}\right\}$ also converge strongly to $q \in$ $F(S) \cap N(A)$. This completes the proof.

The results of Corollary 1 improve the related results in $[14,16,17]$.

Theorem 2. Let E be a reflexive and uniformly convex Banach space which has uniformly Gâteaux differentiable norm and $C$ be a nonempty closed convex subset of $E$ which has normal structure. Let $f: C \rightarrow C$ be a contractive mapping with $k \in[0,1), A$ be a m-accretive operator in $E$ and $S: C \rightarrow C$ be a nonexpansive mapping with $F(S) \cap N(A) \neq \varnothing$. For any $x_{0} \in C$ and $\forall n \geq 0,\left\{x_{n}\right\}$ is generated by

$$
\left\{\begin{array}{l}
y_{n}=\beta_{n}\left(\frac{x_{n}+x_{n+1}}{2}\right)+\left(1-\beta_{n}\right) J_{r_{n}}\left(\frac{x_{n}+x_{n+1}}{2}+e_{n}\right), \\
x_{n+1}=\alpha_{n} f x_{n}+\left(1-\alpha_{n}\right) S y_{n}
\end{array}\right.
$$

where $\left\{\alpha_{n}\right\},\left\{\beta_{n}\right\} \subset(0,1),\left\{e_{n}\right\} \subset E$ and $\left\{r_{n}\right\} \subset(0,1)$ satisfy the following conditions:

(i) $\lim _{n \rightarrow \infty} \alpha_{n}=0, \sum_{n=0}^{\infty} \alpha_{n}=\infty,\left|\alpha_{n}-\alpha_{n-1}\right|=o\left(\alpha_{n}\right)$;

(ii) $\sum_{n=1}^{\infty}\left|\beta_{n}-\beta_{n-1}\right|<\infty$;

(iii) $\lim _{n \rightarrow \infty} r_{n}=r, \sum_{n=1}^{\infty}\left|r_{n}-r_{n-1}\right|<\infty$;

(iv) $\sum_{n=1}^{\infty}\left\|e_{n}\right\|<\infty$.

Then $\left\{x_{n}\right\}$ and $\left\{y_{n}\right\}$ converge strongly to $q \in F(S) \cap N(A)$, where $q$ is the unique solution of the variational inequality $\left\langle(I-f) q, J_{\varphi}(q-p)\right\rangle \leq 0, \forall p \in F(S) \cap N(A)$.

Proof. The proof is split into eleven steps.

Step 1: Show that $\left\{x_{n}\right\}$ and $\left\{y_{n}\right\}$ are bounded.

Take $p \in F(S) \cap N(A)$, then we have

$$
\begin{aligned}
\left\|y_{n}-p\right\| & \leq \beta_{n}\left\|\frac{x_{n}+x_{n+1}}{2}-p\right\|+\left(1-\beta_{n}\right)\left\|J_{r_{n}}\left(\frac{x_{n}+x_{n+1}}{2}+e_{n}\right)-p\right\| \\
& \leq \beta_{n}\left\|\frac{x_{n}+x_{n+1}}{2}-p\right\|+\left(1-\beta_{n}\right)\left\|\frac{x_{n}+x_{n+1}}{2}-p\right\|+\left(1-\beta_{n}\right)\left\|e_{n}\right\| \\
& \leq \frac{1}{2}\left\|x_{n}-p\right\|+\frac{1}{2}\left\|x_{n+1}-p\right\|+\left\|e_{n}\right\|,
\end{aligned}
$$

and then we get

$$
\begin{aligned}
\left\|x_{n+1}-p\right\| & \leq \alpha_{n}\left\|f x_{n}-p\right\|+\left(1-\alpha_{n}\right)\left\|S y_{n}-p\right\| \\
& \leq k \alpha_{n}\left\|x_{n}-p\right\|+\alpha_{n}\|f p-p\|+\left(1-\alpha_{n}\right)\left\|y_{n}-p\right\| \\
& \leq\left(k \alpha_{n}+\frac{1-\alpha_{n}}{2}\right)\left\|x_{n}-p\right\|+\frac{1-\alpha_{n}}{2}\left\|x_{n+1}-p\right\|+\alpha_{n}\|f p-p\|+\left(1-\alpha_{n}\right)\left\|e_{n}\right\| .
\end{aligned}
$$

It follows that 


$$
\begin{aligned}
\left\|x_{n+1}-p\right\| & \leq\left[1-\frac{2 \alpha_{n}(1-k)}{1+\alpha_{n}}\right]\left\|x_{n}-p\right\|+\frac{2 \alpha_{n}}{1+\alpha_{n}}\|f p-p\|+\frac{2\left(1-\alpha_{n}\right)}{1+\alpha_{n}}\left\|e_{n}\right\| \\
& \leq\left[1-\frac{2 \alpha_{n}(1-k)}{1+\alpha_{n}}\right]\left\|x_{n}-p\right\|+\frac{2 \alpha_{n}(1-k)}{1+\alpha_{n}} \frac{\|f p-p\|}{1-k}+2\left\|e_{n}\right\| \\
& \leq \max \left\{\left\|x_{0}-p\right\|, \frac{\|f p-p\|}{1-k}+2\left\|e_{n}\right\|\right\} .
\end{aligned}
$$

Then $\left\{x_{n}\right\}$ and $\left\{y_{n}\right\}$ are bounded. So $\left\{f x_{n}\right\},\left\{f y_{n}\right\},\left\{S x_{n}\right\},\left\{S y_{n}\right\},\left\{J_{r_{n}} x_{n}\right\}$ and $\left\{J_{r_{n}} y_{n}\right\}$ are also bounded.

Step 2: Show that $\lim _{n \rightarrow \infty}\left\|x_{n+1}-x_{n}\right\|=0$.

From (6), we have

$$
\begin{aligned}
\left\|x_{n+1}-x_{n}\right\| & =\left\|\alpha_{n} f x_{n}+\left(1-\alpha_{n}\right) S y_{n}-\alpha_{n-1} f x_{n-1}-\left(1-\alpha_{n-1}\right) S y_{n-1}\right\| \\
& \leq \alpha_{n}\left\|f x_{n}-f x_{n-1}\right\|+\left|\alpha_{n}-\alpha_{n-1}\right| \cdot\left\|f x_{n-1}-S y_{n-1}\right\|+\left(1-\alpha_{n}\right)\left\|S y_{n}-S y_{n-1}\right\| \\
& \leq k \alpha_{n}\left\|x_{n}-x_{n-1}\right\|+\left|\alpha_{n}-\alpha_{n-1}\right| \cdot\left\|f x_{n-1}-S y_{n-1}\right\|+\left(1-\alpha_{n}\right)\left\|y_{n}-y_{n-1}\right\| .
\end{aligned}
$$

From (6), we have

$$
\begin{aligned}
\left\|y_{n}-y_{n-1}\right\| & \leq \beta_{n}\left\|\frac{x_{n+1}-x_{n-1}}{2}\right\|+\left|\beta_{n}-\beta_{n-1}\right| \cdot\left\|\frac{x_{n-1}+x_{n}}{2}-J_{r_{n-1}}\left(\frac{x_{n-1}+x_{n}}{2}+e_{n-1}\right)\right\| \\
& +\left(1-\beta_{n}\right)\left\|J_{r_{n}}\left(\frac{x_{n}+x_{n+1}}{2}+e_{n}\right)-J_{r_{n-1}}\left(\frac{x_{n-1}+x_{n}}{2}+e_{n-1}\right)\right\| .
\end{aligned}
$$

From Lemma 1, we have

$$
\begin{aligned}
& \left\|J_{r_{n}}\left(\frac{x_{n}+x_{n+1}}{2}+e_{n}\right)-J_{r_{n-1}}\left(\frac{x_{n-1}+x_{n}}{2}+e_{n-1}\right)\right\| \\
= & \left\|J_{r_{n-1}}\left(\frac{r_{n-1}}{r_{n}}\left(\frac{x_{n}+x_{n+1}}{2}+e_{n}\right)+\left(1-\frac{r_{n-1}}{r_{n}}\right) J_{r_{n}}\left(\frac{x_{n}+x_{n+1}}{2}+e_{n}\right)\right)-J_{r_{n-1}}\left(\frac{x_{n-1}+x_{n}}{2}+e_{n-1}\right)\right\| \\
\leq & \left\|\frac{r_{n-1}}{r_{n}}\left(\frac{x_{n}+x_{n+1}}{2}+e_{n}\right)+\left(1-\frac{r_{n-1}}{r_{n}}\right) J_{r_{n}}\left(\frac{x_{n}+x_{n+1}}{2}+e_{n}\right)-\left(\frac{x_{n-1}+x_{n}}{2}+e_{n-1}\right)\right\| \\
\leq & \frac{r_{n-1}}{r_{n}}\left\|\frac{x_{n+1}-x_{n-1}}{2}+e_{n}-e_{n-1}\right\|+\left|1-\frac{r_{n-1}}{r_{n}}\right| \cdot\left\|J_{r_{n}}\left(\frac{x_{n}+x_{n+1}}{2}+e_{n}\right)-\left(\frac{x_{n}+x_{n+1}}{2}+e_{n}\right)\right\| \\
+ & \left|1-\frac{r_{n-1}}{r_{n}}\right| \cdot\left\|\frac{x_{n+1}-x_{n-1}}{2}+e_{n}-e_{n-1}\right\| \\
= & \left\|\frac{x_{n+1}-x_{n-1}}{2}+e_{n}-e_{n-1}\right\|+\left|1-\frac{r_{n-1}}{r_{n}}\right| \cdot\left\|J_{r_{n}}\left(\frac{x_{n}+x_{n+1}}{2}\right)-\left(\frac{x_{n}+x_{n+1}}{2}+e_{n}\right)\right\| .
\end{aligned}
$$

Put (8) and (9) into (7), we get

$$
\begin{aligned}
\left\|x_{n+1}-x_{n}\right\| & \leq k \alpha_{n}\left\|x_{n}-x_{n-1}\right\|+\left|\alpha_{n}-\alpha_{n-1}\right| M_{1}+\left(1-\alpha_{n}\right)\left\|\frac{x_{n+1}-x_{n-1}}{2}\right\| \\
& +\left(1-\alpha_{n}\right)\left|\beta_{n}-\beta_{n-1}\right| M_{3}+\left(1-\alpha_{n}\right)\left(1-\beta_{n}\right)\left\|e_{n}-e_{n-1}\right\| \\
& +\left(1-\alpha_{n}\right)\left(1-\beta_{n}\right)\left|1-\frac{r_{n-1}}{r_{n}}\right| M_{4} \\
& \leq\left(k \alpha_{n}+\frac{1-\alpha_{n}}{2}\right)\left\|x_{n}-x_{n-1}\right\|+\frac{1-\alpha_{n}}{2}\left\|x_{n+1}-x_{n}\right\|+\left|\alpha_{n}-\alpha_{n-1}\right| M_{1} \\
& +\left(1-\alpha_{n}\right)\left|\beta_{n}-\beta_{n-1}\right| M_{3}+\left(1-\alpha_{n}\right)\left(1-\beta_{n}\right)\left\|e_{n}-e_{n-1}\right\| \\
& +\left(1-\alpha_{n}\right)\left(1-\beta_{n}\right)\left|1-\frac{r_{n-1}}{r_{n}}\right| M_{4} .
\end{aligned}
$$

It follows that

$$
\begin{aligned}
\left\|x_{n+1}-x_{n}\right\|= & {\left[1-\frac{2 \alpha_{n}(1-k)}{1+\alpha_{n}}\right]\left\|x_{n}-x_{n-1}\right\|+\frac{2\left|\alpha_{n}-\alpha_{n-1}\right|}{1+\alpha_{n}} M_{1}+\frac{2\left(1-\alpha_{n}\right)}{1+\alpha_{n}}\left|\beta_{n}-\beta_{n-1}\right| M_{3} } \\
& +\frac{2\left(1-\alpha_{n}\right)\left(1-\beta_{n}\right)}{1+\alpha_{n}}\left(\left\|e_{n}\right\|+\left\|e_{n-1}\right\|\right)+\frac{2\left(1-\alpha_{n}\right)\left(1-\beta_{n}\right)\left|r_{n}-r_{n-1}\right|}{\left(1+\alpha_{n}\right) r_{n}} M_{4},
\end{aligned}
$$

where 
$M_{3}=\max \left\|\frac{x_{n}+x_{n+1}}{2}-J_{r_{n}}\left(\frac{x_{n}+x_{n+1}}{2}+e_{n}\right)\right\|, M_{4}=\max \left\|J_{r_{n}}\left(\frac{x_{n}+x_{n+1}}{2}+e_{n}\right)-\left(\frac{x_{n}+x_{n+1}}{2}+e_{n}\right)\right\|$.

Take $t_{n}=\frac{2 \alpha_{n}(1-k)}{1+\alpha_{n}}$, then $t_{n}>\alpha_{n}(1-k)$. From $\sum_{n=0}^{\infty} \alpha_{n}=\infty$, so $\sum_{n=0}^{\infty} t_{n}=\infty$.

Take $b_{n}=\frac{2\left|\alpha_{n}-\alpha_{n-1}\right|}{1+\alpha_{n}} M_{1}$, then $\frac{b_{n}}{t_{n}}=\frac{\left|\alpha_{n}-\alpha_{n-1}\right| M_{1}}{\alpha_{n}(1-k)}$. From $\left|\alpha_{n}-\alpha_{n-1}\right|=o\left(\alpha_{n}\right)$, so $b_{n}=o\left(t_{n}\right)$.

Take

$c_{n}=\frac{2\left(1-\alpha_{n}\right)}{1+\alpha_{n}}\left|\beta_{n}-\beta_{n-1}\right| M_{3}+\frac{2\left(1-\alpha_{n}\right)\left(1-\beta_{n}\right)}{1+\alpha_{n}}\left(\left\|e_{n}\right\|+\left\|e_{n-1}\right\|\right)+\frac{2\left(1-\alpha_{n}\right)\left(1-\beta_{n}\right)\left|r_{n}-r_{n-1}\right|}{\left(1+\alpha_{n}\right) r_{n}} M_{4}$,

then $c_{n}<2\left|\beta_{n}-\beta_{n-1}\right| M_{3}+2\left(\left\|e_{n}\right\|+\left\|e_{n-1}\right\|\right)+\frac{2\left|r_{n}-r_{n-1}\right| M_{4}}{r+\varepsilon}(\forall \varepsilon>0)$.

From $\sum_{n=1}^{\infty}\left|\beta_{n}-\beta_{n-1}\right|<\infty, \sum_{n=1}^{\infty}\left\|e_{n}\right\|<\infty, \lim _{n \rightarrow \infty} r_{n}=r$ and $\sum_{n=1}^{\infty}\left|r_{n}-r_{n-1}\right|<\infty$, so $\sum_{n=1}^{\infty} c_{n}<\infty$.

From Lemma 2, we get $\lim _{n \rightarrow \infty}\left\|x_{n+1}-x_{n}\right\|=0$.

Step 3: Show that $\lim _{n \rightarrow \infty}\left\|x_{n}-J_{r_{n}} x_{n}\right\|=0$.

Because $\|\cdot\|^{2}$ is convex function and (1), so we have

$$
\begin{aligned}
& \left\|x_{n+1}-p\right\|^{2} \leq \alpha_{n}\left\|f x_{n}-p\right\|^{2}+\left(1-\alpha_{n}\right)\left\|S y_{n}-p\right\|^{2} \\
& \leq \alpha_{n}\left\|f x_{n}-p\right\|^{2}+\left(1-\alpha_{n}\right)\left\|y_{n}-p\right\|^{2} \\
& \leq \alpha_{n}\left\|f x_{n}-p\right\|^{2}+\left(1-\alpha_{n}\right) \beta_{n}\left\|\frac{x_{n}+x_{n+1}}{2}-p\right\|^{2} \\
& +\left(1-\alpha_{n}\right)\left(1-\beta_{n}\right)\left\|J_{r_{n}}\left(\frac{x_{n}+x_{n+1}}{2}+e_{n}\right)-p\right\|^{2} \\
& =\alpha_{n}\left\|f x_{n}-p\right\|^{2}+\left(1-\alpha_{n}\right)\left[\beta_{n}\left\|\frac{x_{n}+x_{n+1}}{2}-p\right\|^{2}\right. \\
& \left.+\left(1-\beta_{n}\right)\left\|J_{\frac{r_{n}}{2}}\left(\frac{1}{2}\left(\frac{x_{n}+x_{n+1}}{2}+e_{n}\right)+\frac{1}{2} J_{r_{n}}\left(\frac{x_{n}+x_{n+1}}{2}+e_{n}\right)\right)-p\right\|^{2}\right] \\
& \leq \alpha_{n}\left\|f x_{n}-p\right\|^{2}+\left(1-\alpha_{n}\right) \beta_{n}\left\|\frac{x_{n}+x_{n+1}}{2}-p\right\|^{2} \\
& +\left(1-\alpha_{n}\right)\left(1-\beta_{n}\right)\left\|\frac{1}{2}\left(\frac{x_{n}+x_{n+1}}{2}+e_{n}-p\right)+\frac{1}{2}\left(J_{r_{n}}\left(\frac{x_{n}+x_{n+1}}{2}+e_{n}\right)-p\right)\right\|^{2} \\
& \leq \alpha_{n}\left\|f x_{n}-p\right\|^{2}+\left(1-\alpha_{n}\right) \beta_{n}\left\|\frac{x_{n}+x_{n+1}}{2}-p\right\|^{2} \\
& +\left(1-\alpha_{n}\right)\left(1-\beta_{n}\right)\left\|\frac{x_{n}+x_{n+1}}{2}+e_{n}-p\right\|^{2} \\
& -\frac{\left(1-\alpha_{n}\right)\left(1-\beta_{n}\right)}{4} g\left(\left\|\frac{x_{n}+x_{n+1}}{2}+e_{n}-J_{r_{n}}\left(\frac{x_{n}+x_{n+1}}{2}+e_{n}\right)\right\|\right) \\
& \leq \alpha_{n}\left\|f x_{n}-p\right\|^{2}+\left(1-\alpha_{n}\right) \beta_{n}\left\|\frac{x_{n}+x_{n+1}}{2}-p\right\|^{2} \\
& +\left(1-\alpha_{n}\right)\left(1-\beta_{n}\right)\left\|\frac{x_{n}+x_{n+1}}{2}+e_{n}-p\right\|^{2} \\
& -\frac{\left(1-\alpha_{n}\right)\left(1-\beta_{n}\right)}{4} g\left(\left\|\frac{x_{n}+x_{n+1}}{2}+e_{n}-J_{r_{n}}\left(\frac{x_{n}+x_{n+1}}{2}+e_{n}\right)\right\|\right) \\
& \leq \alpha_{n}\left\|f x_{n}-p\right\|^{2}+\left(1-\alpha_{n}\right) \beta_{n}\left\|\frac{x_{n}+x_{n+1}}{2}-p\right\|^{22} \\
& +\left(1-\alpha_{n}\right)\left(1-\beta_{n}\right)\left\|\frac{x_{n}+x_{n+1}}{2}+e_{n}-p\right\| \\
& +\left(1-\alpha_{n}\right)\left(1-\beta_{n}\right)\left(\left\|\frac{x_{n}+x_{n+1}}{2}-p\right\|^{2}+2\left\langle e_{n}, J\left(\frac{x_{n}+x_{n+1}}{2}+e_{n}-p\right)\right\rangle\right) \\
& -\frac{\left(1-\alpha_{n}\right)\left(1-\beta_{n}\right)}{4} g\left(\left\|\frac{x_{n}+x_{n+1}}{2}+e_{n}-J_{r_{n}}\left(\frac{x_{n}+x_{n+1}}{2}+e_{n}\right)\right\|\right) \\
& =\alpha_{n}\left\|f x_{n}-p\right\|^{2}+\left(1-\alpha_{n}\right)\left\|\frac{x_{n}+x_{n+1}}{2}-p\right\|^{2} \\
& +2\left(1-\alpha_{n}\right)\left(1-\beta_{n}\right)\left\langle e_{n}, J\left(\frac{x_{n}+x_{n+1}}{2}+e_{n}-p\right)\right\rangle \\
& -\frac{\left(1-\alpha_{n}\right)\left(1-\beta_{n}\right)}{4} g\left(\left\|\frac{x_{n}+x_{n+1}}{2}+e_{n}-J_{r_{n}}\left(\frac{x_{n}+x_{n+1}}{2}+e_{n}\right)\right\|\right) \\
& \leq \alpha_{n}\left\|f x_{n}-p\right\|^{2}+\frac{1-\alpha_{n}}{2}\left\|x_{n}-p\right\|^{2}+\frac{1-\alpha_{n}}{2}\left\|x_{n+1}-p\right\|^{2} \\
& +2\left(1-\alpha_{n}\right)\left(1-\beta_{n}\right)\left\langle e_{n}, J\left(\frac{x_{n}+x_{n+1}}{2}+e_{n}-p\right)\right\rangle \\
& -\frac{\left(1-\alpha_{n}\right)\left(1-\beta_{n}\right)}{4} g\left(\left\|\frac{x_{n}+x_{n+1}}{2}+e_{n}-J_{r_{n}}\left(\frac{x_{n}+x_{n+1}}{2}+e_{n}\right)\right\|\right) \text {. }
\end{aligned}
$$


It follows that

$$
\begin{aligned}
\left\|x_{n+1}-p\right\|^{2} & \leq \frac{1-\alpha_{n}}{1+\alpha_{n}}\left\|x_{n}-p\right\|^{2}+\frac{2 \alpha_{n}}{1+\alpha_{n}}\left\|f x_{n}-p\right\|^{2}+\frac{\left(1-\alpha_{n}\right)\left(1-\beta_{n}\right)}{1+\alpha_{n}}\left\|e_{n}\right\| \cdot\left\|\frac{x_{n}+x_{n+1}}{2}+e_{n}-p\right\| \\
& -\frac{\left(1-\alpha_{n}\right)\left(1-\beta_{n}\right)}{4} g\left(\left\|\frac{x_{n}+x_{n+1}}{2}+e_{n}-J_{r_{n}}\left(\frac{x_{n}+x_{n+1}}{2}+e_{n}\right)\right\|\right) \\
& \leq\left\|x_{n}-p\right\|^{2}+\frac{2 \alpha_{n}}{1+\alpha_{n}}\left\|f x_{n}-p\right\|^{2}+\frac{\left(1-\alpha_{n}\right)\left(1-\beta_{n}\right)}{1+\alpha_{n}}\left\|e_{n}\right\| \cdot\left\|\frac{x_{n}+x_{n+1}}{2}+e_{n}-p\right\| \\
& -\frac{\left(1-\alpha_{n}\right)\left(1-\beta_{n}\right)}{4} g\left(\left\|\frac{x_{n}+x_{n+1}}{2}+e_{n}-J_{r_{n}}\left(\frac{x_{n}+x_{n+1}}{2}+e_{n}\right)\right\|\right) .
\end{aligned}
$$

Then we have

$$
\begin{aligned}
& \frac{\left(1-\alpha_{n}\right)\left(1-\beta_{n}\right)}{4} g\left(\left\|\frac{x_{n}+x_{n+1}}{2}+e_{n}-J_{r_{n}}\left(\frac{x_{n}+x_{n+1}}{2}+e_{n}\right)\right\|\right)-\frac{2 \alpha_{n}}{1+\alpha_{n}}\left\|f x_{n}-p\right\|^{2}- \\
& \frac{\left(1-\alpha_{n}\right)\left(1-\beta_{n}\right)}{1+\alpha_{n}}\left\|e_{n}\right\| \cdot\left\|\frac{x_{n}+x_{n+1}}{2}+e_{n}-p\right\| \leq\left\|x_{n}-p\right\|^{2}-\left\|x_{n+1}-p\right\|^{2} .
\end{aligned}
$$

If $\frac{\left(1-\alpha_{n}\right)\left(1-\beta_{n}\right)}{4} g\left(\left\|\frac{x_{n}+x_{n+1}}{2}+e_{n}-J_{r_{n}}\left(\frac{x_{n}+x_{n+1}}{2}+e_{n}\right)\right\|\right) \leq \frac{2 \alpha_{n}}{1+\alpha_{n}}\left\|f x_{n}-p\right\|^{2}+\frac{\left(1-\alpha_{n}\right)\left(1-\beta_{n}\right)}{1+\alpha_{n}}\left\|e_{n}\right\|$. $\left\|\frac{x_{n}+x_{n+1}}{2}+e_{n}-p\right\|$, so from $\lim _{n \rightarrow \infty} \alpha_{n}=0$, step 1 and $\sum_{n=0}^{\infty}\left\|e_{n}\right\|<\infty$, we get $\lim _{n \rightarrow \infty} g\left(\left\|\frac{x_{n}+x_{n+1}}{2}+e_{n}-J_{r_{n}}\left(\frac{x_{n}+x_{n+1}}{2}+e_{n}\right)\right\|\right)=0$.

If $\frac{\left(1-\alpha_{n}\right)\left(1-\beta_{n}\right)}{4} g\left(\left\|\frac{x_{n}+x_{n+1}}{2}+e_{n}-J_{r_{n}}\left(\frac{x_{n}+x_{n+1}}{2}+e_{n}\right)\right\|\right) \geq \frac{2 \alpha_{n}}{1+\alpha_{n}}\left\|f x_{n}-p\right\|^{2}+\frac{\left(1-\alpha_{n}\right)\left(1-\beta_{n}\right)}{1+\alpha_{n}}\left\|e_{n}\right\| \cdot$ $\left\|\frac{x_{n}+x_{n+1}}{2}+e_{n}-p\right\|$, so

$$
\begin{aligned}
& \sum_{n=0}^{N}\left[\frac{\left(1-\alpha_{n}\right)\left(1-\beta_{n}\right)}{4} g\left(\left\|\frac{x_{n}+x_{n+1}}{2}+e_{n}-J_{r_{n}}\left(\frac{x_{n}+x_{n+1}}{2}+e_{n}\right)\right\|\right)-\frac{2 \alpha_{n}}{1+\alpha_{n}}\left\|f x_{n}-p\right\|^{2}-\right. \\
& \left.\frac{\left(1-\alpha_{n}\right)\left(1-\beta_{n}\right)}{1+\alpha_{n}}\left\|e_{n}\right\| \cdot\left\|\frac{x_{n}+x_{n+1}}{2}+e_{n}-p\right\|\right] \leq\left\|x_{0}-p\right\|^{2}-\left\|x_{N+1}-p\right\|^{2} \leq\left\|x_{0}-p\right\|^{2} .
\end{aligned}
$$

Then

$$
\begin{aligned}
& \sum_{n=0}^{\infty}\left[\frac{\left(1-\alpha_{n}\right)\left(1-\beta_{n}\right)}{4} g\left(\left\|\frac{x_{n}+x_{n+1}}{2}+e_{n}-J_{r_{n}}\left(\frac{x_{n}+x_{n+1}}{2}+e_{n}\right)\right\|\right)-\frac{2 \alpha_{n}}{1+\alpha_{n}}\left\|f x_{n}-p\right\|^{2}-\right. \\
& \left.\frac{\left(1-\alpha_{n}\right)\left(1-\beta_{n}\right)}{1+\alpha_{n}}\left\|e_{n}\right\| \cdot\left\|\frac{x_{n}+x_{n+1}}{2}+e_{n}-p\right\|\right]<\infty .
\end{aligned}
$$

So we get

$$
\begin{aligned}
& \lim _{n \rightarrow \infty}\left[\frac{\left(1-\alpha_{n}\right)\left(1-\beta_{n}\right)}{4} g\left(\left\|\frac{x_{n}+x_{n+1}}{2}+e_{n}-J_{r_{n}}\left(\frac{x_{n}+x_{n+1}}{2}+e_{n}\right)\right\|\right)-\frac{2 \alpha_{n}}{1+\alpha_{n}}\left\|f x_{n}-p\right\|^{2}-\right. \\
& \left.\frac{\left(1-\alpha_{n}\right)\left(1-\beta_{n}\right)}{1+\alpha_{n}}\left\|e_{n}\right\| \cdot\left\|\frac{x_{n}+x_{n+1}}{2}+e_{n}-p\right\|\right]=0
\end{aligned}
$$

and then $\lim _{n \rightarrow \infty} g\left(\left\|\frac{x_{n}+x_{n+1}}{2}+e_{n}-J_{r_{n}}\left(\frac{x_{n}+x_{n+1}}{2}+e_{n}\right)\right\|\right)=0$.

From the property of $g$, so we get $\lim _{n \rightarrow \infty}\left\|\frac{x_{n}+x_{n+1}}{2}+e_{n}-J_{r_{n}}\left(\frac{x_{n}+x_{n+1}}{2}+e_{n}\right)\right\|=0$.

We also have

$$
\begin{aligned}
\left\|x_{n}-J_{r_{n}} x_{n}\right\| & \leq\left\|x_{n}-\left(\frac{x_{n}+x_{n+1}}{2}+e_{n}\right)\right\|+\left\|\frac{x_{n}+x_{n+1}}{2}+e_{n}-J_{r_{n}}\left(\frac{x_{n}+x_{n+1}}{2}+e_{n}\right)\right\| \\
& +\left\|J_{r_{n}}\left(\frac{x_{n}+x_{n+1}}{2}+e_{n}\right)-J_{r_{n}} x_{n}\right\| \\
& \leq 2\left\|x_{n}-\left(\frac{x_{n}+x_{n+1}}{2}+e_{n}\right)\right\|+\left\|\frac{x_{n}+x_{n+1}}{2}+e_{n}-J_{r_{n}}\left(\frac{x_{n}+x_{n+1}}{2}+e_{n}\right)\right\| \\
& \leq\left\|x_{n}-x_{n+1}\right\|+2\left\|e_{n}\right\|+\left\|\frac{x_{n}+x_{n+1}}{2}+e_{n}-J_{r_{n}}\left(\frac{x_{n}+x_{n+1}}{2}+e_{n}\right)\right\| .
\end{aligned}
$$

Then from step 2 and $\sum_{n=0}^{\infty}\left\|e_{n}\right\|<\infty$, we get $\lim _{n \rightarrow \infty}\left\|x_{n}-J_{r_{n}} x_{n}\right\|=0$. 
Step 4: Show that $\lim _{n \rightarrow \infty}\left\|y_{n}-S y_{n}\right\|=0$.

$$
\begin{aligned}
\left\|y_{n}-S y_{n}\right\| & \leq \beta_{n}\left\|\frac{x_{n}+x_{n+1}}{2}-S y_{n}\right\|+\left(1-\beta_{n}\right)\left\|J_{r_{n}}\left(\frac{x_{n}+x_{n+1}}{2}+e_{n}\right)-S y_{n}\right\| \\
& \leq\left\|\frac{x_{n}+x_{n+1}}{2}-S y_{n}\right\|+\left(1-\beta_{n}\right)\left\|e_{n}\right\| \\
& +\left(1-\beta_{n}\right)\left\|J_{r_{n}}\left(\frac{x_{n}+x_{n+1}}{2}+e_{n}\right)-\left(\frac{x_{n}+x_{n+1}}{2}+e_{n}\right)\right\| \\
& \leq\left\|\frac{x_{n}+x_{n+1}}{2}-x_{n+1}\right\|+\left\|x_{n+1}-S y_{n}\right\|+\left(1-\beta_{n}\right)\left\|e_{n}\right\| \\
& +\left(1-\beta_{n}\right)\left\|J_{r_{n}}\left(\frac{x_{n}+x_{n+1}}{2}+e_{n}\right)-\left(\frac{x_{n}+x_{n+1}}{2}+e_{n}\right)\right\| \\
& \leq \frac{1}{2}\left\|x_{n}-x_{n+1}\right\|+\alpha_{n}\left\|f x_{n}-S y_{n}\right\|+\left(1-\beta_{n}\right)\left\|e_{n}\right\| \\
& +\left(1-\beta_{n}\right)\left\|J_{r_{n}}\left(\frac{x_{n}+x_{n+1}}{2}+e_{n}\right)-\left(\frac{x_{n}+x_{n+1}}{2}+e_{n}\right)\right\| .
\end{aligned}
$$

From step 1, step 2, step $3, \lim _{n \rightarrow \infty} \alpha_{n}=0$ and $\sum_{n=0}^{\infty}\left\|e_{n}\right\|<\infty$, we get $\lim _{n \rightarrow \infty}\left\|y_{n}-S y_{n}\right\|=0$.

Step 5: Show that $\lim _{n \rightarrow \infty}\left\|x_{n}-y_{n}\right\|=0$.

$$
\begin{aligned}
\left\|x_{n}-y_{n}\right\| & \leq\left\|x_{n}-x_{n+1}\right\|+\left\|x_{n+1}-S y_{n}\right\|+\left\|S y_{n}-y_{n}\right\| \\
& =\left\|x_{n}-x_{n+1}\right\|+\alpha_{n}\left\|f x_{n}-S y_{n}\right\|+\left\|S y_{n}-y_{n}\right\| .
\end{aligned}
$$

From step1, step 2, step 4 and $\lim _{n \rightarrow \infty} \alpha_{n}=0$, we get $\lim _{n \rightarrow \infty}\left\|x_{n}-y_{n}\right\|=0$.

Step 6: Show that $\lim _{n \rightarrow \infty}\left\|y_{n}-J_{r_{n}} y_{n}\right\|=0$.

$$
\begin{aligned}
\left\|y_{n}-J_{r_{n}} y_{n}\right\| & \leq\left\|y_{n}-x_{n}\right\|+\left\|x_{n}-J_{r_{n}} x_{n}\right\|+\left\|J_{r_{n}} x_{n}-J_{r_{n}} y_{n}\right\| \\
& \leq 2\left\|y_{n}-x_{n}\right\|+\left\|x_{n}-J_{r_{n}} x_{n}\right\| .
\end{aligned}
$$

From steps 3 and 5, we get $\lim _{n \rightarrow \infty}\left\|y_{n}-J_{r_{n}} y_{n}\right\|=0$.

Step 7: Show that $\lim _{n \rightarrow \infty}\left\|x_{n}-S x_{n}\right\|=0$.

$$
\begin{aligned}
\left\|x_{n}-S x_{n}\right\| & \leq\left\|x_{n}-y_{n}\right\|+\left\|y_{n}-S y_{n}\right\|+\left\|S y_{n}-S x_{n}\right\| \\
& \leq 2\left\|x_{n}-y_{n}\right\|+\left\|y_{n}-S y_{n}\right\| .
\end{aligned}
$$

From steps 4 and 5, we get $\lim _{n \rightarrow \infty}\left\|x_{n}-S x_{n}\right\|=0$.

Step 8: Show that $\lim _{n \rightarrow \infty}\left\|y_{n}-J_{r} y_{n}\right\|=0$.

$$
\begin{aligned}
\left\|y_{n}-J_{r} y_{n}\right\| & \leq\left\|y_{n}-J_{r_{n}} y_{n}\right\|+\left\|J_{r_{n}} y_{n}-J_{r} y_{n}\right\| \\
& =\left\|y_{n}-J_{r_{n}} y_{n}\right\|+\left\|J_{r}\left(\frac{r}{r_{n}} y_{n}+\left(1-\frac{r}{r_{n}}\right) J_{r_{n}} y_{n}\right)-J_{r} y_{n}\right\| \\
& \leq\left\|y_{n}-J_{r_{n}} y_{n}\right\|+\left|1-\frac{r}{r_{n}}\right| \cdot\left\|J_{r_{n}} y_{n}-y_{n}\right\| .
\end{aligned}
$$

From step 6 and $\lim _{n \rightarrow \infty} r_{n}=r$, we get $\lim _{n \rightarrow \infty}\left\|y_{n}-J_{r} y_{n}\right\|=0$.

Step 9: Show that $\lim _{n \rightarrow \infty}\left\|x_{n}-J_{r} x_{n}\right\|=0$.

$$
\begin{aligned}
\left\|x_{n}-J_{r} x_{n}\right\| & \leq\left\|x_{n}-y_{n}\right\|+\left\|y_{n}-J_{r} y_{n}\right\|+\left\|J_{r} y_{n}-J_{r} x_{n}\right\| \\
& \leq 2\left\|x_{n}-y_{n}\right\|+\left\|y_{n}-J_{r} y_{n}\right\| .
\end{aligned}
$$

From steps 5 and 8 , we get $\lim _{n \rightarrow \infty}\left\|x_{n}-J_{r} x_{n}\right\|=0$. 
Step 10: Show that $\limsup _{n \rightarrow \infty}\left\langle q-f q, J\left(q-x_{n}\right)\right\rangle=0$.

From Theorem 1, we have that $\left\{x_{t}\right\}$ converges strongly to $q \in P_{F(S) \cap N(A)} f q$, which is also the unique solution of the variational inequality $\langle q-f q, J(q-p)\rangle \leq 0, \forall p \in F(S) \cap N(A)$.

We have

$$
\begin{aligned}
\left\|x_{t}-x_{n}\right\|^{2} & =(1-t)\left\langle S x_{t}-S x_{n}+S x_{n}-x_{n}, J\left(x_{t}-x_{n}\right)\right\rangle+t\left\langle f x_{t}-x_{t}+x_{t}-x_{n}, J\left(x_{t}-x_{n}\right)\right\rangle \\
& \leq(1-t)\left\|x_{t}-x_{n}\right\|^{2}+(1-t)\left\|S x_{n}-x_{n}\right\| \cdot\left\|x_{t}-x_{n}\right\|+t\left\|x_{t}-x_{n}\right\|^{2}+t\left\langle f x_{t}-x_{t}, J\left(x_{t}-x_{n}\right)\right\rangle \\
& =\left\|x_{t}-x_{n}\right\|^{2}+(1-t)\left\|S x_{n}-x_{n}\right\| \cdot\left\|x_{t}-x_{n}\right\|+t\left\|x_{t}-x_{n}\right\|^{2}+t\left\langle f x_{t}-x_{t}, J\left(x_{t}-x_{n}\right)\right\rangle .
\end{aligned}
$$

It follows that $\left\langle f x_{t}-x_{t}, J\left(x_{t}-x_{n}\right)\right\rangle \leq \frac{1-t}{t}\left\|S x_{n}-x_{n}\right\| \cdot\left\|x_{t}-x_{n}\right\|$. From step 1 and step 7 , we get $\limsup _{n \rightarrow \infty}\left\langle q-f q, J\left(q-x_{n}\right)\right\rangle=0$.

Step 11: Show that $\lim _{n \rightarrow \infty}\left\|x_{n}-q\right\|=0$.

From Lemma 4, we have

$$
\begin{aligned}
\left\|x_{n+1}-q\right\|^{2} & \leq\left(1-\alpha_{n}\right)^{2}\left\|S y_{n}-q\right\|^{2}+2 \alpha_{n}\left\langle f x_{n}-q, J\left(x_{n+1}-q\right)\right\rangle \\
& \leq\left(1-\alpha_{n}\right)^{2}\left\|y_{n}-q\right\|^{2}+2 k \alpha_{n}\left\|x_{n}-q\right\| \cdot\left\|x_{n+1}-q\right\|+2 \alpha_{n}\left\langle f q-q, J\left(x_{n+1}-q\right)\right\rangle \\
& \leq\left(1-\alpha_{n}\right)^{2}\left(\frac{1}{2}\left\|x_{n}-q\right\|+\frac{1}{2}\left\|x_{n+1}-q\right\|+\left\|e_{n}\right\|\right)^{2}+2 k \alpha_{n}\left\|x_{n}-q\right\| \cdot\left\|x_{n+1}-q\right\| \\
& +2 \alpha_{n}\left\langle f q-q, J\left(x_{n+1}-q\right)\right\rangle \\
& =\left(\frac{1-\alpha_{n}}{2}\right)^{2}\left\|x_{n}-q\right\|^{2}+\left(\frac{1-\alpha_{n}}{2}\right)^{2}\left\|x_{n+1}-q\right\|^{2}+\left(1-\alpha_{n}\right)^{2}\left\|e_{n}\right\|^{2} \\
& +\frac{\left(1-\alpha_{n}\right)^{2}}{2}\left\|x_{n}-q\right\| \cdot\left\|x_{n+1}-q\right\|+\left(1-\alpha_{n}\right)^{2}\left\|x_{n}-q\right\| \cdot\left\|e_{n}\right\| \\
& +\left(1-\alpha_{n}\right)^{2}\left\|x_{n+1}-q\right\| \cdot\left\|e_{n}\right\|+2 k \alpha_{n}\left\|x_{n}-q\right\| \cdot\left\|x_{n+1}-q\right\| \\
& +2 \alpha_{n}\left\langle f q-q, J\left(x_{n+1}-q\right)\right\rangle \\
& \leq\left(\frac{1-\alpha_{n}}{2}\right)^{2}\left\|x_{n}-q\right\|^{2}+\left(\frac{1-\alpha_{n}}{2}\right)^{2}\left\|x_{n+1}-q\right\|^{2} \\
& +\left[\frac{\left(1-\alpha_{n}\right)^{2}}{4}+k \alpha_{n}\right]\left(\left\|x_{n}-q\right\|^{2}+\left\|x_{n+1}-q\right\|^{2}\right)+\left(1-\alpha_{n}\right)^{2}\left\|e_{n}\right\|^{2} \\
& +\left(1-\alpha_{n}\right)^{2}\left\|e_{n}\right\|\left(\left\|x_{n}-q\right\|+\left\|x_{n+1}-q\right\|\right)+2 \alpha_{n}\left\langle f q-q, J\left(x_{n+1}-q\right)\right\rangle .
\end{aligned}
$$

It follows that

$$
\begin{aligned}
\left\|x_{n+1}-q\right\|^{2} & \leq \frac{\left(\frac{1-\alpha_{n}}{2}\right)^{2}+\frac{\left(1-\alpha_{n}\right)^{2}}{4}+k \alpha_{n}}{1-\left[\left(\frac{1-\alpha_{n}}{2}\right)^{2}+\frac{\left(1-\alpha_{n}\right)^{2}}{4}+k \alpha_{n}\right]}\left\|x_{n}-q\right\|^{2} \\
& +\frac{2 \alpha_{n}}{1-\left[\left(\frac{1-\alpha_{n}}{2}\right)^{2}+\frac{\left(1-\alpha_{n}\right)^{2}}{4}+k \alpha_{n}\right]}\left\langle f q-q, J\left(x_{n+1}-q\right)\right\rangle \\
& +\frac{\left(1-\alpha_{n}\right)^{2}\left\|e_{n}\right\|}{1-\left[\left(\frac{1-\alpha_{n}}{2}\right)^{2}+\frac{\left(1-\alpha_{n}\right)^{2}}{4}+k \alpha_{n}\right]}\left(\left\|x_{n}-q\right\|+\left\|x_{n+1}-q\right\|+\left\|e_{n}\right\|\right) \\
& =\left[1-\frac{1-2\left(\frac{\left(1-\alpha_{n}\right)^{2}}{2}+k \alpha_{n}\right)}{1-\left(\frac{\left(1-\alpha_{n}\right)^{2}}{2}+k \alpha_{n}\right)}\right]\left\|x_{n}-q\right\|^{2} \\
& +\frac{2 \alpha_{n}}{1-\left[\frac{\left(1-\alpha_{n}\right)^{2}}{2}+k \alpha_{n}\right]}\left\langle f q-q, J\left(x_{n+1}-q\right)\right\rangle \\
& +\frac{\left(1-\alpha_{n}\right)^{2}\left\|e_{n}\right\|}{1-\left[\frac{\left(1-\alpha_{n}\right)^{2}}{2}+k \alpha_{n}\right]}\left(\left\|x_{n}-q\right\|+\left\|x_{n+1}-q\right\|+\left\|e_{n}\right\|\right) .
\end{aligned}
$$


Take $t_{n}=\frac{1-2\left(\frac{\left(1-\alpha_{n}\right)^{2}}{2}+k \alpha_{n}\right)}{1-\left(\frac{\left(1-\alpha_{n}\right)^{2}}{2}+k \alpha_{n}\right)}$. From $\lim _{n \rightarrow \infty} \alpha_{n}=0$, we have

$$
t_{n} \geq 1-2\left(\frac{\left(1-\alpha_{n}\right)^{2}}{2}+k \alpha_{n}\right)=\alpha_{n}\left(2-2 k-\alpha_{n}\right) \geq \alpha_{n}(2-2 k-\varepsilon)(\forall \varepsilon>0)
$$

From $\sum_{n=0}^{\infty} \alpha_{n}=\infty$, we get $\sum_{n=0}^{\infty} t_{n}=\infty$.

Take $b_{n}=\frac{2 \alpha_{n}}{1-\left[\frac{\left(1-\alpha_{n}\right)^{2}}{2}+k \alpha_{n}\right]}\left\langle f q-q, J\left(x_{n+1}-q\right)\right\rangle$, then we have

$$
\frac{b_{n}}{t_{n}}=\frac{2 \alpha_{n}\left\langle f q-q, J\left(x_{n+1}-q\right)\right\rangle}{1-2\left[\frac{\left(1-\alpha_{n}\right)^{2}}{2}+k \alpha_{n}\right]}=\frac{2\left\langle f q-q, J\left(x_{n+1}-q\right)\right\rangle}{2-2 k-\alpha_{n}} .
$$

From $\lim _{n \rightarrow \infty} \alpha_{n}=0$ and step 10, we get $b_{n}=o\left(t_{n}\right)$.

Take $c_{n}=\frac{\left(1-\alpha_{n}\right)^{2}\left\|e_{n}\right\|}{1-\left[\frac{\left(1-\alpha_{n}\right)^{2}}{2}+k \alpha_{n}\right]}\left(\left\|x_{n}-q\right\|+\left\|x_{n+1}-q\right\|+\left\|e_{n}\right\|\right)$, then

$$
c_{n}=\frac{2\left(1-\alpha_{n}\right)^{2}\left\|e_{n}\right\|}{1+\alpha_{n}\left(2-2 k-\alpha_{n}\right)}\left(\left\|x_{n}-q\right\|+\left\|x_{n+1}-q\right\|+\left\|e_{n}\right\|\right) .
$$

From $k \in(0,1)$ and $\alpha_{n} \in(0,1)$, we get $\alpha_{n}\left(2-2 k-\alpha_{n}\right)>0$, and then $c_{n}<$ $2\left(\left\|x_{n}-q\right\|+\left\|x_{n+1}-q\right\|+\left\|e_{n}\right\|\right)\left\|e_{n}\right\|$. From $\sum_{n=0}^{\infty}\left\|e_{n}\right\|<\infty$, we get $\sum_{n=0}^{\infty} c_{n}<\infty$.

From Lemma 2, we get $\lim _{n \rightarrow \infty}\left\|x_{n}-q\right\|=0$. This completes the proof.

The results of Theorem 2 improve the related results in [15-17]. For example, the results of Theorem 2 is can obtain the related results in [15-17]; the rate of convergence and computational accuracy is better than their in [15-17].

\section{Numerical Examples}

We give four numerical examples to support the main results.

Example 1. Let $R$ be the real line with Euclidean norm, $f: R \rightarrow R$ be defined by $f(x)=\frac{x}{6}, S: R \rightarrow R$ be defined by $S(x)=\frac{x}{4}$ and $J_{r_{n}} x=\frac{r_{n} x}{2}$. So $F(T)=\{0\}$. Let $\alpha_{n}=\frac{1}{n}, \beta_{n}=\frac{1}{n}$ and $r_{n}=1-\frac{1}{n}$, then they satisfy the conditions of Theorem 1. $\left\{x_{n}\right\}$ is generated by (2). From Theorem 1, we can obtain $\left\{x_{n}\right\}$ converges strongly to 0 .

Next, we simplify the form of (2) and get

$$
x_{n+1}=\frac{3-9 n+9 n^{2}+3 n^{3}-14 n^{4}}{-3+9 n-9 n^{2}-51 n^{3}+6 n^{4}} x_{n} .
$$

Next, we take $x_{1}=1$ into (10). Finally, we get the following numerical results in Figure 1. 


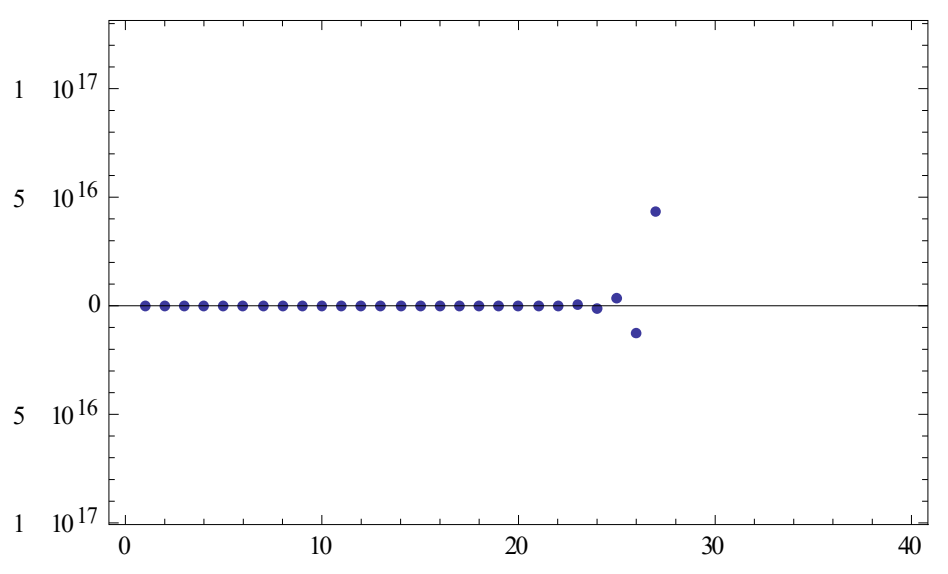

Figure 1. Numerical results.

Example 2. Let $R$ be the real line with Euclidean norm, $f: R \rightarrow R$ be defined by $f(x)=\frac{x}{6}, S: R \rightarrow R$ be defined by $S(x)=\frac{x}{4}$ and $J_{r_{n}} x=\frac{r_{n} x}{2}$. So $F(T)=\{0\}$. Let $\alpha_{n}=\frac{1}{n}, \beta_{n}=\frac{1}{n}, r_{n}=1-\frac{1}{n}$ and $e_{n}=\frac{1}{n^{2}}$, then they satisfy the conditions of Theorem 2. $\left\{x_{n}\right\}$ is generated by (6). From Theorem 2, we can obtain $\left\{x_{n}\right\}$ converges strongly to 0 .

Next, we simplify the form of (6) and get

$$
x_{n+1}=-\frac{2(n-1)^{2} n^{4}}{-1+3 n-3 n^{2}-17 n^{3}+2 n^{4}} x_{n} .
$$

Next, we take $x_{1}=1$ into (11). Finally, we get the following numerical results in Figure 2.

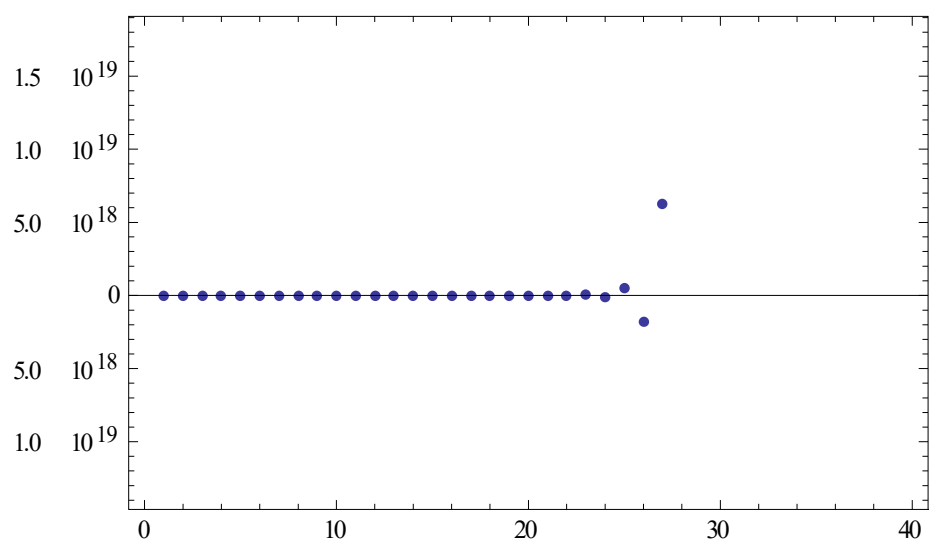

Figure 2. Numerical results.

Example 3. Let $\langle\cdot, \cdot\rangle: R^{3} \times R^{3} \rightarrow R$ be the inner product and defined by

$$
\langle x, y\rangle=x_{1} y_{1}+x_{2} y_{2}+x_{2} y_{3}
$$

Let $\|\cdot\|: R^{3} \rightarrow R$ be the usual norm and defined by $\|x\|=\sqrt{x_{1}^{2}+x_{2}^{2}+x_{3}^{2}}$ for any $x=\left(x_{1}, x_{2}, x_{3}\right)$.

For any $x \in R^{3}$, let $f: R^{3} \rightarrow R^{3}$ be defined by $f(x)=\frac{x}{6}, S: R^{3} \rightarrow R^{3}$ be defined by $S(x)=\frac{x}{4}$ and $J_{r_{n}} x=\frac{r_{n} x}{2}$. So $F(T)=\{0\}$. Let $\alpha_{n}=\frac{1}{n}, \beta_{n}=\frac{1}{n}$ and $r_{n}=1-\frac{1}{n}$, then they satisfy the conditions of Theorem 1. $\left\{x_{n}\right\}$ is generated by (2). From Theorem 1, we can obtain $\left\{x_{n}\right\}$ converges strongly to 0. 
Next, we simplify the form of (2) and get

$$
x_{n+1}=\frac{3-9 n+9 n^{2}+3 n^{3}-14 n^{4}}{-3+9 n-9 n^{2}-51 n^{3}+6 n^{4}} x_{n} .
$$

Next, we take $x_{1}=(1,2,3)$ into (12). Finally, we get the following numerical results in Figure 3.

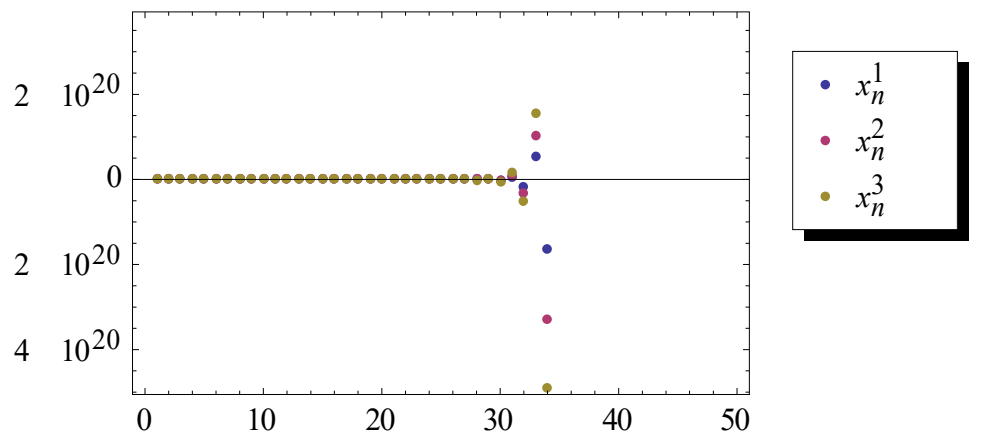

Figure 3. Numerical results.

Example 4. Let $\langle\cdot, \cdot\rangle: R^{3} \times R^{3} \rightarrow R$ be the inner product and defined by

$$
\langle x, y\rangle=x_{1} y_{1}+x_{2} y_{2}+x_{2} y_{3}
$$

Let $\|\cdot\|: R^{3} \rightarrow R$ be the usual norm and defined by $\|x\|=\sqrt{x_{1}^{2}+x_{2}^{2}+x_{3}^{2}}$ for any $x=\left(x_{1}, x_{2}, x_{3}\right)$.

For any $x \in R^{3}$, let $f: R^{3} \rightarrow R^{3}$ be defined by $f(x)=\frac{x}{6}, S: R^{3} \rightarrow R^{3}$ be defined by $S(x)=\frac{x}{4}$ and $J_{r_{n}} x=\frac{r_{n} x}{2}$. So $F(T)=\{0\}$. Let $\alpha_{n}=\frac{1}{n}, \beta_{n}=\frac{1}{n}, r_{n}=1-\frac{1}{n}$ and $e_{n}=\frac{1}{n^{2}}$, then they satisfy the conditions of Theorem 2. $\left\{x_{n}\right\}$ is generated by (6). From Theorem 2, we can obtain $\left\{x_{n}\right\}$ converges strongly to 0 .

Next, we simplify the form of (6) and get

$$
x_{n+1}=-\frac{2(n-1)^{2} n^{4}}{-1+3 n-3 n^{2}-17 n^{3}+2 n^{4}} x_{n} .
$$

Next, we take $x_{1}=(1,10,100)$ into (13). Finally, we get the following numerical results in Figure 4 .

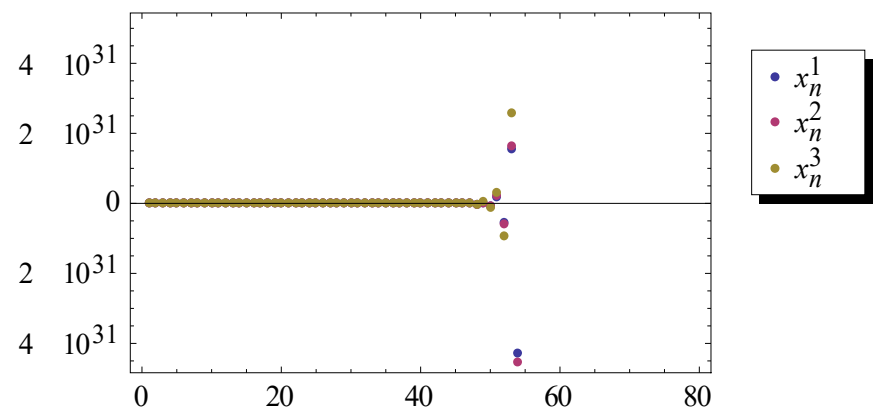

Figure 4. Numerical results.

Author Contributions: Conceptualization, H.Z.; Funding acquisition, Y.Q.; Methodology, Y.S.

Funding: This research was funded by [National Natural Science Foundation of Hebei Province] grant number [E2016209304] and the APC was funded by [National Natural Science Foundation of Hebei Province].

Acknowledgments: This research is supported by the National Natural Science Foundation of Hebei Province (No. E2016209304).

Conflicts of Interest: The authors declare no conflict of interest. 


\section{References}

1. Chang, S.S.; Lee, H.W.J.; Chan, C.K. Strong convergence theorems by viscosity approximation methods for accretive mappings and nonexpansive mappings. J. Appl. Math. Inform. 2009, 27, 59-68.

2. Jung, J.S.; Cho, Y.J.; Zhou, H.Y. Iterative processes with mixed errors for nonlinear equationswith perturbed m-accretive operators in Banach spaces. Appl. Math. Comput. 2002, 133, 389-406.

3. Moudafi, A. Viscosity approximation methods for fixed points problems. J. Math. Anal. Appl. 2000, 241, 46-55. [CrossRef]

4. Qin, X.L.; Cho, S.Y.; Wang, L. Iterative algorithms with errors for zero points of m-accretive Operators. Fixed Point Theory Appl. 2013, 2013, 148. [CrossRef]

5. Reich, S. Approximating zeros of accretive operators. Proc. Am. Math. Soc. 1975, 51, 381-384. [CrossRef]

6. Reich, S. On fixed point theorems obtained from existence theorems for differential equations. J. Math. Anal. Appl. 1976, 54, 26-36. [CrossRef]

7. Auzinger, W.; Frank, R. Asymptotic error expansions for stiff equations: An analysis for the implicit midpoint and trapezoidal rules in the strongly stiff case. Numer. Math. 1989, 56, 469-499. [CrossRef]

8. Bader, G.; Deuflhard, P. A semi-implicit mid-point rule for stiff systems of ordinary differential equations. Numer. Math. 1983, 41, 373-398. [CrossRef]

9. Deuflhard, P. Recent progress in extrapolation methods for ordinary differential equations. SIAM Rev. 1985, 27, 505-535. [CrossRef]

10. Schneider, C. Analysis of the linearly implicit mid-point rule for differential-algebra equations. Electron. Trans. Numer. Anal. 1993, 1, 1-10.

11. Somalia, S. Implicit midpoint rule to the nonlinear degenerate boundary value problems. Int. J. Comput. Math. 2002, 79, 327-332. [CrossRef]

12. Van Veldhuxzen, M. Asymptotic expansions of the global error for the implicit midpoint rule (stiff case). Computing 1984, 33, 185-192. [CrossRef]

13. Jung, J.S. Strong convergence of viscosity approximation methods for finding zeros of accretive operators in Banach spaces. Nonlinear Anal. 2010, 72, 449-459. [CrossRef]

14. Jung, J.S. Strong convergence of an iterative algorithm for accretive operators and nonexpansive mappings. J. Nonlinear Sci. Appl. 2016, 9, 2394-2409. [CrossRef]

15. Li, D.F. On nonexpansive and accretive operators in Banach spaces. J. Nonlinear Sci. Appl. 2017, 10, 3437-3446. [CrossRef]

16. Xu, H.K.; Alghamdi, M.A.; Shahzad, N. The viscosity technique for the implicit midpoint rule of nonexpansive mappings in Hilbert spaces. Fixed Point Theory Appl. 2015, 2015, 41. [CrossRef]

17. Luo, P.; Cai, G.; Shehu, Y. The viscosity iterative algorithms for the implicit midpoint rule of nonexpansive mappings in uniformly smooth Banach spaces. J. Inequal. Appl. 2017, 2017, 154. [CrossRef] [PubMed]

18. Reich, S. On the asymptotic behavior of nonlinear semigroups and the range of accretive operators. J. Math. Anal. Appl. 1981, 79, 113-126. [CrossRef]

19. Barbu, V. Nonlinear semigroups and differential equations in Banach spaces. Editura Academiei Republicii Socialiste Romania 1976, 2, 1-6.

20. Liu, L.S. Ishikawa and Mann iterative process with errors for nonlinear strongly accretive mappings in Banach spaces. J. Math. Anal. Appl. 1995, 194, 114-125. [CrossRef]

21. Reich, S. Strong convergence theorems for resolvents of accretive operators in Banach spaces. J. Math. Anal. Appl. 1980, 75, 287-292. [CrossRef]

(C) 2018 by the authors. Licensee MDPI, Basel, Switzerland. This article is an open access article distributed under the terms and conditions of the Creative Commons Attribution (CC BY) license (http:/ / creativecommons.org/licenses/by/4.0/). 